\title{
Vortical structures and development of laminar flow over convergent-divergent riblets
}

DOI:

$10.1063 / 1.5027522$

\section{Document Version}

Accepted author manuscript

Link to publication record in Manchester Research Explorer

\section{Citation for published version (APA):}

Xu, F., Zhong, S., \& Zhang, S. (2018). Vortical structures and development of laminar flow over convergentdivergent riblets. Physics of Fluids, 30(5), [051901]. https://doi.org/10.1063/1.5027522

\section{Published in:}

Physics of Fluids

\section{Citing this paper}

Please note that where the full-text provided on Manchester Research Explorer is the Author Accepted Manuscript or Proof version this may differ from the final Published version. If citing, it is advised that you check and use the publisher's definitive version.

\section{General rights}

Copyright and moral rights for the publications made accessible in the Research Explorer are retained by the authors and/or other copyright owners and it is a condition of accessing publications that users recognise and abide by the legal requirements associated with these rights.

\section{Takedown policy}

If you believe that this document breaches copyright please refer to the University of Manchester's Takedown Procedures [http://man.ac.uk/04Y6Bo] or contact uml.scholarlycommunications@manchester.ac.uk providing relevant details, so we can investigate your claim.

\section{OPEN ACCESS}




\title{
Vortical structures and development of laminar flow over convergent-divergent riblets
}

\author{
Fang $\mathrm{Xu},{ }^{1}$, a) Shan Zhong, ${ }^{1}$ and Shanying Zhang ${ }^{1}$ \\ School of Mechanical, Aerospace and Civil Engineering, University of Manchester, Manchester, M13 9PL, \\ United Kingdom
}

(Dated: 12 April 2018)

In this work, the development of a laminar boundary layer over a rectangular convergent-divergent riblet section with a finite streamwise length is studied experimentally using dye visualization and PIV in a water flume. The flow topology over this highly directional spanwise roughness is established from this study. It is shown that convergent-divergent riblets generate a spanwise flow above the riblets from the diverging line towards the adjacent converging line. This consequently leads to the formation of a weak recirculating secondary flow in cross-stream planes across the boundary layer that creates a downwash motion over the diverging line and an upwash motion over the converging line. It is found that the fluid inside the riblet valley follows a helicoidal path and it also interacts with the crossflow boundary layer hence playing a key role in determining the structure of the secondary flow across the boundary layer. The impact of riblet wavelength on vortical structures is also revealed for the first time. A larger riblet wavelength is seen to produce a stronger upwash/downwash and hence a more intense secondary flow as well as a stronger deceleration effect on the crossflow. Furthermore, the streamwise development of the flow over the riblet section can be divided into a developing stage followed by a developed stage. In the developing stage, the magnitude of induced streamwise velocity and vorticity over the converging line continue to increase, whereas in the developed stage the values of these parameters remain essentially unchanged.

\section{NOMENCLATURE}

$\begin{array}{ll}x & {[m]} \\ y & {[m]} \\ z & {[m]} \\ u & {\left[\mathrm{~ms}^{-1}\right]} \\ v & {\left[\mathrm{~ms}^{-1}\right]} \\ w & {\left[\mathrm{~ms}^{-1}\right]} \\ U_{\infty} & {\left[\mathrm{ms}^{-1}\right]} \\ l & {[\mathrm{~m}]} \\ \nu & {\left[\mathrm{m}^{2} \mathrm{~s}^{-1}\right.} \\ \operatorname{Re}_{l} & {[1]} \\ \omega_{x} & {\left[\mathrm{~s}^{-1}\right]} \\ \omega_{y} & {\left[\mathrm{~s}^{-1}\right]} \\ \omega_{z} & {\left[\mathrm{~s}^{-1}\right]} \\ T u & {[1]} \\ \delta & {[\mathrm{m}]} \\ \theta & {[\mathrm{m}]} \\ \operatorname{Re}_{\theta} & {[1]} \\ s & {[\mathrm{~m}]} \\ s_{x} & {[\mathrm{~m}]} \\ s_{z} & {[\mathrm{~m}]} \\ h & {[\mathrm{~m}]} \\ h_{p} & {[\mathrm{~m}]} \\ \Lambda & {[\mathrm{m}]} \\ L & {[\mathrm{~m}]} \\ W & {[\mathrm{~m}]} \\ \gamma & {[1]} \\ \lambda_{c i} & {\left[\mathrm{~s}^{-1}\right]} \\ & \end{array}$

streamwise coordinate

wall-normal coordinate

spanwise coordinate

streamwise velocity

wall-normal velocity

spanwise velocity

freestream velocity

streamwise coordinate of the center of

riblet section

kinematic viscosity

Reynolds number based on $l$

vorticity in $y$ - $z$ plane

vorticity in $x$ - $z$ plane

vorticity in $x$ - $y$ plane

turbulence intensity

boundary layer thickness $\left(99 \% U_{\infty}\right)$

boundary layer momentum thickness

momentum thickness Reynolds number

riblet spacing

riblet spacing projected in $x$ direction

riblet spacing projected in $z$ direction

riblet height

riblet protrusion height

riblet wavelength

length of riblet section

width of riblet section

riblet yaw angle

swirling strength $\left\langle\lambda_{c i}\right\rangle\left[s^{-1}\right]$

$A_{\Delta U} \quad\left[m s^{-1}\right]$

$W_{\Delta U}[m]$

$A_{\omega_{y}} \quad\left[s^{-1}\right]$

TIU $\left[m^{2} s^{-1}\right]$

signed swirling strength

amplitude of induced velocity

width of converging/diverging region

amplitude of vorticity

total induced velocity

PIV particle image velocimetry

CL converging line

$\mathrm{DL}$ diverging line

FP flat plate

\section{INTRODUCTION}

Longitudinal (streamwise) riblets have been studied since $1980 \mathrm{~s}^{1,2}$ and a large volume of research work has been undertaken in laminar boundary layers, ${ }^{3-5}$ transitional boundary layers, ${ }^{6}$ and turbulent boundary layers. ${ }^{7-9}$ The drag reduction performance and the control mechanism of longitudinal riblets are wellunderstood. It has been reported that longitudinal riblets are capable of delivering a reduction of surface friction drag around $10 \%$ in a turbulent boundary layer. ${ }^{9,10}$ The drag reduction mechanism of longitudinal riblets is attributed to the damping of crossflow fluctuations ${ }^{11-13}$ or the uplift of turbulent streamwise vortices above the riblet valley. ${ }^{14-17}$. There are some comprehensive reviews of the advances in research on longitudinal riblets. ${ }^{18-20}$

Convergent-divergent riblets studied in this paper are a new type of directional surface roughness patterns which begins to attract research attention more recently. For this type of surface patterns, sections of riblets with a negative yaw angle and a positive yaw angle with respect to the freestream direction are arranged in an alterna- 
tive manner in the spanwise direction, see Fig. 1. The patterns of convergent-divergent riblets are inspired by nature. Converging riblet patterns are found upstream of the sensory receptors of sharks, whereas the diverging patterns are observed upstream of the hearing sensors of these animals. ${ }^{21}$ Diverging riblet patterns (also called herringbone riblets) also appear on the secondary flight feathers of different species of birds. ${ }^{22}$

Koeltzsch et al. ${ }^{21}$ studied the flow in a pipe whose inner surface was fitted with riblet films arranged obliquely, with $-45^{\circ}$ yaw angle in one half of the pipe circumference and $+45^{\circ}$ yaw angle in the other. They observed an increase in the local streamwise velocity and a decrease in turbulent fluctuations over the diverging riblet pattern, and the opposite effect was found over the converging riblet pattern. Inspired by this finding, a series of experimental studies on convergent-divergent riblets has been carried out in a flat plate turbulent boundary layer at the University of Melbourne. Similar results to those reported by Koeltzsch et al. ${ }^{21}$ were also obtained by Nugroho et al. ${ }^{23}$ Using a cross-wire, Nugroho et al. ${ }^{24}$ revealed the presence of a large-scale counter-rotating rol1 mode in the time-averaged flow field in cross-stream planes. Their finding was also confirmed and extended by Kevin et al. ${ }^{25}$ with their stereoscopic PIV measurements. In accordance to the sense of rotation of the counter-rotating roll mode, the fluid in the region over the diverging line appears to be directed towards the wall (downwash), whereas the fluid in the region over the converging line is seen to be directed away from the wall (upwash).

There is an inherent difference between the vortical structures induced by convergent-divergent riblets and those induced by longitudinal (streamwise) riblets. The spanwise length scale of the vortical structures induced by longitudinal riblets has the same order as that of the riblet spacing $s .^{7,11}$ The effect of longitudinal riblets is known to be confined in the vicinity of the ribleted surface $^{4,26}$ via suppression of streamwise vortical structures in the near-wall region. ${ }^{6,27}$ In contrast, the spanwise length scale of the vortical structures produced by the convergent-divergent riblets is comparable to the riblet wavelength $\Lambda$ or the boundary layer thickness $\delta,{ }^{25}$ which could be one or two orders of magnitude larger than that of the riblet spacing $s$. As such, the whole boundary layer can be affected. ${ }^{23}$

The global effects of convergent-divergent riblets on a flow field have also been explored by some researchers. Chen et al. ${ }^{22}$ carried out an experiment in a fullydeveloped turbulent pipe flow and reported that pipes covered with spanwise arrays of convergent-divergent riblets with diminishing heights towards their edges yield a drag reduction. With an optimal yaw angle of $30^{\circ}$ and an optimal riblet height of 20 viscous wall units, a $21 \%$ drag reduction was reported, which is significantly larger than what longitudinal riblets could ever offer $(\sim 8 \%$ in Walsh ${ }^{10}, 9.9 \%$ in Bechert et al. $\left.{ }^{9}\right)$. In a linear cascade experiment, Liu et al. ${ }^{28}$ demonstrated that herringbone riblets fitted on blade suction surfaces were capable of reducing pressure losses by suppressing flow separation at low Reynolds numbers. Such flow control benefits have been attributed to the large scale streamwise vortical structures produced by convergent-divergent riblets. It is envisaged that convergent-divergent riblets could have a broad engineering application prospect including surface friction drag reduction, suppression of flow separation and heat transfer augmentation.

In contrast to the large quantity of studies on longitudinal riblets, there are only a very limited number of studies on convergent-divergent riblets, see Table I. There has been no research investigating how the flow initially develops itself over these surface patterns. Such a study will be meaningful to practical applications, such as turbine blades, for which, due to their short streamwise extent, knowledge of the early stage of flow development over the riblet section is essential to maximize the effect of the surface roughness to the flow. It is known that the convergent-divergent riblets act to divert some flow near the surface away from the converging region, leading to the generation of spanwise flow and the counter-rotating vortex pairs. However, there is a lack of understanding as to how the small-scale flow motion inside riblet channels contributes to the establishment of the large-scale motion. Furthermore, in most of the existing experimental studies the wavelength of the convergent-divergent riblet$\mathrm{s}$ is chosen arbitrarily and hence it is not clear how the wavelength affects the strength and the characteristics of the induced secondary flow.

Therefore, in this paper the development of a flat plate laminar flow over a section of surface covered with convergent-divergent riblets of different wavelengths is investigated in a water flume. Dye visualization technique is firstly used to visualize the small-scale fluid motion inside riblet valleys and the way that it interacts with the crossflow over riblet crests. Mono PIV is then used to investigate the impact of the riblets on the boundary layer development over the converging region and diverging region respectively. Finally, stereoscopic PIV is employed to examine the vortical structures in the cross-stream plane. From the analysis of these results, the flow topology over convergent-divergent riblets is established and the effect of riblet wavelength on the strength and characteristics of the secondary flow is understood for the first time.

The use of a laminar boundary layer instead of a turbulent one in this study enables a clearer flow topology and hence allows a better understanding of the associated flow physics to be established without the interference of complex turbulent events. Considering the fact that convergent-divergent riblets are observed on both shark skin and bird feathers, such surface patterns are likely to find applications in both low Reynolds number flows where laminar flow exists and high Reynolds number flows where the flow is fully turbulent. Therefore, the finding from our study could directly benefit practical applications in low Reynolds number flows. Furthermore, 


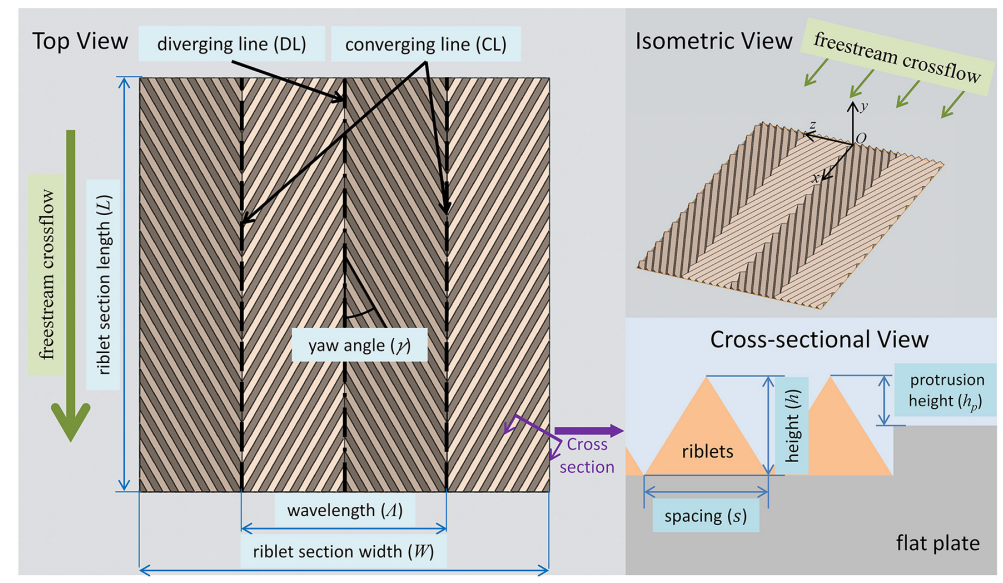

FIG. 1. Schematic diagram of convergent-divergent riblets parameters. Converging line $(C L)$ and diverging line $(D L)$ are defined intuitively from the arrangement of the riblets relative to the freestream direction. The converging/diverging region is referred to the region close to the converging/diverging line.

understanding the impact of convergent-divergent riblets on a laminar boundary layer forms a logical first step towards understanding their effect on a turbulent boundary layer.

\section{EXPERIMENTAL SETTING}

In this section, the experimental settings of the water flume, convergent-divergent riblets, the dye visualization system and PIV systems are described, and the nondimensionalization of the coordinate system is provided.

\section{A. WATER FLUME}

Our experiments are conducted in a water flume at the University of Manchester, which is depicted in Fig. 2a. The water flume is $3.64 \mathrm{~m}$ long and it has a $0.305 \mathrm{~m} \times$ $0.305 \mathrm{~m}$ cross-section. Both the side walls and the bottom floor of the test section are made of transparent glass to provide an optical access required for the PIV system. The fluid in the flume is driven by a centrifugal pump which draws water from the holding tank into the entry tank. To ensure a uniform flow with low turbulence level in the test section, the fluid is made to pass through a set of guiding vanes and a honeycomb before it goes through four metal screens arranged in a decreasing order of mesh size. The freestream velocity and water level in the test section are controlled by changing the rotating speed of the centrifugal pump with a digital inverter and an opening of the adjustable permeable grate installed at the outlet of the flume.

An aluminum flat plate $(1380 \mathrm{~mm}$ long, $300 \mathrm{~mm}$ wide, $6 \mathrm{~mm}$ thick) is installed horizontally in the water flume with its test surface facing downward. In this setup, the use of a $1: 5$ superelliptical leading edge together with a negative inclination angle $\left(-0.3^{\circ}\right)$ of the flat plate is found to be effective in ensuring that an attached laminar boundary layer is obtained right from the leading edge. ${ }^{33}$ A small gap is left between the flat plate and each side wall of the test section to restrain the growth of side wall boundary layers along the length of the flat plate. The stereoscopic PIV data of the baseline flow field (not shown here) confirms the $2 D$ characteristic$\mathrm{s}$ of the boundary layer flow across the width of the convergent-divergent riblet section. In this experimen$\mathrm{t}$, the freestream velocity is set as $U_{\infty}=0.1 \mathrm{~m} / \mathrm{s}$ and the turbulence intensity in the freestream crossflow is about $0.2 \%$. The Reynolds number based on the streamwise distance of the measurement station $(l=715 \mathrm{~mm})$ from the leading edge of flat plate $\left(R e_{l}=U_{\infty} l / \nu\right)$ is $7.15 \times 10^{4}$, and the Reynolds number based on the momentum thickness $\left(\operatorname{Re}_{\theta}=U_{\infty} \theta / \nu\right)$ is 165. As shown in Fig. 2b, the velocity profile measured at this location fits well with the Blasius solution obtained assuming that the boundary layer starts from the leading edge, confirming that the boundary layer under investigation is close to a zeropressure-gradient laminar boundary layer.

\section{B. CONVERGENT-DIVERGENT RIBLETS}

The disc plate containing the convergent-divergent riblets is made with clear resin using $3 D$ printing technique. It has a diameter $D=132 \mathrm{~mm}$ and it can be flush-mounted into the test plate as an insert. The center of this disc is located at $715 \mathrm{~mm}$ downstream of the leading edge of the test plate. The cross-sectional shape of the riblets is triangular with $s=3 \mathrm{~mm}$ and $h=2.4 \mathrm{~mm}$, see Fig. 1 . The protrusion height $\left(h_{p}\right)$ of convergent-divergent riblets is $1.2 \mathrm{~mm}$, which indicates that the smooth wall levels with half of the riblet height $\left(h_{p}=h / 2\right)$. The blockage ratio, i.e., the protrusion height of the riblets to the flat plate boundary layer thickness $\left(h_{p} / \delta_{F P}\right)$, is about $9 \%$ at the start of the riblet sec- 


\begin{tabular}{|c|c|c|c|c|c|c|c|c|}
\hline Research & Model & Fluid & Section $(m)$ & Measure & $R e$ & $h\left(h^{+}\right)(\mu m)$ & $s\left(s^{+}\right)(\mu m)$ & $\gamma(\operatorname{deg})$ \\
\hline Koeltzsch et al. ${ }^{21}$ & pipe & air & $0.2(L), 0.07(R)$ & hot-wire & $5.2 \times 10^{5}$ & $152(19)$ & $152(19)$ & 45 \\
\hline Nugroho et al. ${ }^{29}$ & plate & air & $0.515(L), 0.296(W)$ & hot-wire & 1900 & $500(17)$ & $675(23)$ & 30 \\
\hline Nugroho et al. ${ }^{30}$ & plate & air & $0.515(L), 0.296(W)$ & hot-wire & $1300 / 2300$ & $500(13 / 24)$ & $675(18 / 32)$ & 10 \\
\hline Nugroho et al. ${ }^{23}$ & plate & air & $1 \sim 4(L), 0.296(W)$ & hot-wire & $771 \sim 3120$ & $500(6.7 \sim 24.4)$ & $675(9 \sim 33)$ & $10 / 30$ \\
\hline Chen et al. ${ }^{31}$ & pipe & water & $0.4(L), 0.026(R)$ & pressure gauge & $1.4 \sim 3.7 \times 10^{5}$ & $60(19)$ & 100 & 30 \\
\hline Chen et al. ${ }^{22}$ & pipe & water & $0.6(L), 0.026(R)$ & pressure gauge & $1.1 \sim 4.7 \times 10^{5}$ & $60(19)$ & 100 & $0 \sim 60$ \\
\hline Kevin et al. ${ }^{32}$ & plate & air & $0.515(L), 0.296(W)$ & mono PIV & - & $500(18)$ & $675(24)$ & 20 \\
\hline Kevin et al. ${ }^{25}$ & plate & air & $4(L), 0.296(W)$ & stereo PIV & 3900 & $500(18)$ & $675(24)$ & 20 \\
\hline
\end{tabular}

TABLE I. A summary of experimental parameters in existing studies. In pipe flows, Reynolds number $R e_{D}=\bar{U} D / \nu$, where $D$ is the hydraulic diameter. For flat plate flows, $R e_{\tau}=u_{\tau} L / \nu$, where $u_{\tau}$ is the friction velocity.
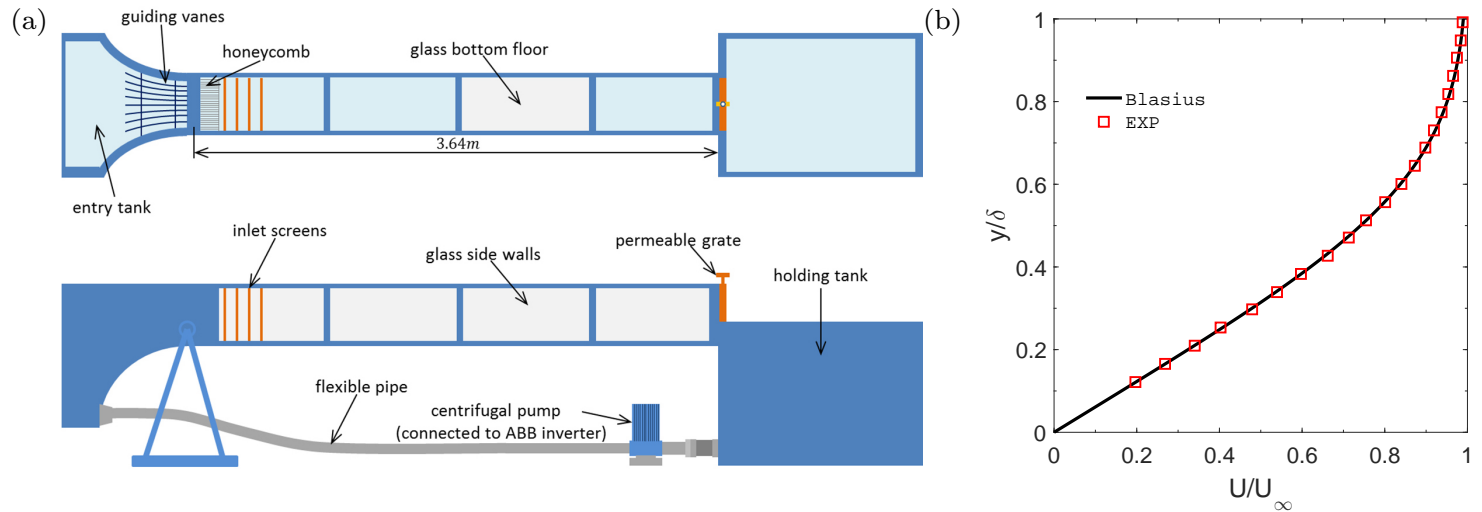

FIG. 2. (a) Schematic diagram of the water flume in the top view (upper) and the side view (lower). (b) Comparison of the measured streamwise velocity profile in the experimental section at $x=0.715 \mathrm{~mm}$ with the Blasius solution.

tion. With both the layer thickness and the particles diameter being set as $25 \mu \mathrm{m}$, the resolution of $3 D$ printing ( $\sim 1 \%$ of the riblet spacing or the riblet height) is considered excellent for the present setup. The angle between tilted riblets and the freestream direction is the yaw angle $(\gamma)$. The optimal yaw angle of $\gamma=30^{\circ}$ identified by Chen et al. ${ }^{22}$ in their turbulent pipe flow experiment is adopted in this experiment. The overall length and width of the riblet section is $L=90 \mathrm{~mm}$ by $W=90 \mathrm{~mm}$. Riblet sections with a wavelength of $30 \mathrm{~mm}, 36 \mathrm{~mm}$ and $45 \mathrm{~mm}$ are tested in this experiment. The total number of riblet wavelengths accommodated on the disc plate is hence 3.0, 2.5 and 2.0 respectively.

\section{NONDIMENSIONALIZATION OF COORDINATE SYSTEM}

The coordinate system is shown in Figs. 1 and 3c, with axes $x, y$ and $z$ representing the streamwise, wall-normal and spanwise directions respectively, and the corresponding velocity components are $u, v$ and $w$ respectively. $x=0$ is located at the beginning of the convergentdivergent riblet section, which is $670 \mathrm{~mm}$ downstream of the leading edge of the flat plate. $y=0$ is chosen to coincide with the smooth surface (level with the mid height of the riblets), and $z=0$ is located at the converging/diverging line closest to the middle in the spanwise direction.

To provide nondimensional length scales, the three coordinate axes are normalized as follows. The streamwise coordinate $x$ is normalized by the riblet spacing projected in the longitudinal plane $\left(s_{x}=s / \sin \gamma=3 \mathrm{~mm} / \sin 30^{\circ}=\right.$ $6 \mathrm{~mm})$. Thus, $x / s_{x}$ indicates the number of riblets in the streamwise direction. The wall-normal coordinate $y$ is normalized by the local boundary layer thickness of the flat plate $\left(\delta_{F P}\right)$. The spanwise coordinate $z$ is normalized by the wavelength of convergent-divergent riblets $(\Lambda)$.

\section{DYE VISUALIZATION SYSTEM}

The setup of the dye visualization experimental system includes dye injection, illumination and image acquisition. In the present experiment, the dye is fed from a $3 D$ printed cavity through five rubber tubes, which are connected to the five dye injection orifices on the disc plate located at a small distance of $10 \mathrm{~mm}$ upstream of the riblet section. The dye flow rate through each rubber tube can be individually controlled using tube clips. The top side of the disc plate with convergent-divergent riblets remains translucent, whereas the bottom side is paint- 
ed white to increase the contrast of dye images. The remaining part of the disc plate is painted black.

During the experiment, the riblet section is illuminated using four white LED light sources through the glass side walls of the flume. To avoid producing shadows of dye streaklines on the riblet surface, lighting from the top is not used. The dye visualization images are captured using a Canon D70 SLR camera fitted with a Canon EF$\mathrm{S} 18-135 \mathrm{~mm}$ F3.5-5.6 IS lens. The camera is operated using a remote control mode to minimize its movement. The aperture of the camera lens is set as 11.0 in the side view and 5.6 in the top view. The smaller aperture used for the side view enables a larger depth of view across the span.

\section{E. PARTICLE IMAGE VELOCIMETRY SYSTEM}

The PIV system from TSI used in this experiment is composed of a New Wave Solo-PIV 120 laser generator, a laser pulse synchronizer (Model 610036) and two CCD cameras. The Solo-PIV 120 laser generator is capable of firing two laser pluses in a rapid succession with a maximum power of $135 \mathrm{~mJ}$ and a pulse width of $3 \sim 5 \mathrm{~ns}$ at a repetition rate up to $15 \mathrm{~Hz}$. The CCD cameras have a $12-$ bit intensity dynamic range and a maximum resolution of $2352 \times 1768$ (4 Mega) pixels. The cameras are capable of recording images at a frame rate of up to $16 \mathrm{~Hz}$. A $532 \pm 10 \mathrm{~nm}$ bandpass filter is installed on the Nikon AF $50 \mathrm{~mm} f / 1.8 D$ camera lens to further improve the image quality by reducing the intensity of the background light. The Dantec Dynamics HGS-10 (Hollow Glass Spheres) particles with a mean diameter of $10 \mu \mathrm{m}$ and density of $1.1 \times 10^{3} \mathrm{~kg} / \mathrm{m}^{3}$ are used as the seeding particles. The settling velocity of these particles is $5 \times 10^{-6} \mathrm{~m} / \mathrm{s}$, ensuring that particles follow the motion of the fluid with high fidelity. To reduce the laser reflection and improve the quality of PIV images near the wall, the riblet section is coated with a matt black paint.

The layouts of the mono PIV measurements in the longitudinal $(x-y)$ plane and the wall-parallel $(x-z)$ plane are shown in Figs. 3a and $3 \mathrm{~b}$ respectively. The aperture of the lens is set as $f / 4.0$. The time interval between two laser pulses is chosen to be $6 \mathrm{~ms}$ for the longitudinal plane and $2.5 \mathrm{~ms}$ for the wall-parallel plane. The distance from the laser head to the measurement section is about $1 \mathrm{~m}$. The width of the laser light sheet is around $1 \mathrm{~mm}$. A total of 1250 image pairs are captured at an acquisition rate of $2 \mathrm{~Hz}$, which is more than sufficient to ensure the convergence of the time-averaged flow field characteristics. In order to capture the flow field across the entire disc plate and beyond, mono PIV measurements are taken on two overlapping longitudinal planes separately, as illustrated in Fig. 4, and the data in these two planes are then merged together at the post-processing stage. In the wall-parallel plane, the center of the laser light sheet is approximately $3 \mathrm{~mm}$ from the smooth wall surface.

The PIV image pairs are processed using TSI InSight
$4 G$ software to obtain the velocity vector field. The first interrogation area is $96 \times 32$ pixels, and the final interrogation area is $32 \times 32$ pixels with $50 \%$ overlap. In the longitudinal plane, the field of view is $85 \mathrm{~mm} \times 64 \mathrm{~mm}$, yielding a velocity vector field with a spatial resolution of $0.58 \mathrm{~mm} \times 0.58 \mathrm{~mm}$. In the wall-parallel plane, the field of view is $138 \mathrm{~mm} \times 104 \mathrm{~mm}$, resulting in a spatial resolution of $0.94 \mathrm{~mm} \times 0.94 \mathrm{~mm}$. In the longitudinal plane, due to the high disparity in the displacements of particles between near-wall flow and the freestream, the image deformation algorithm is applied during post-processing. The measurement uncertainties in velocity components are estimated using the Peak Ratio (PR) method, which includes factors such as the particle seeding density, the pre-processing of images, the pixel displacement and other possible sources in determining the uncertainty. ${ }^{34}$ For an instantaneous flow field, the expanded uncertainty in velocity components, i.e., $95.4 \%$ possibility of having the true value within the uncertainty bound, is estimated to be $0.43 \% U_{\infty}$ in the longitudinal plane and $0.91 \% U_{\infty}$ in the wall-parallel plane. In the averaged flow field of 1250 image pairs, the expanded uncertainty in velocity components is $0.05 \% U_{\infty}$ in the longitudinal plane and $0.28 \% U_{\infty}$ in the wall-parallel plane.

In stereoscopic PIV, the cross-stream $(y-z)$ plane is located at $x=12.5 s_{x}$. One 4 Mega pixel CCD camera and a water-filled prism are installed at each side of the water flume, as shown in Fig. 3c. The locations of the cameras are adjusted according to the Scheimpflug principle. The camera calibration is undertaken using a TSI $200 \mathrm{~mm} \times 200 \mathrm{~mm}$ calibration target plate, and the perspectives of cameras are optimized by the ensembleaveraged dewrapped velocity field. The time interval between the two laser pulses is set as $2.5 \mathrm{~ms}$, and $1250 \mathrm{im}$ age pairs are captured at low acquisition rate of $1 \mathrm{~Hz}$. The first interrogation area of $64 \times 24$ pixels and the final interrogation area of $24 \times 16$ pixels with $50 \%$ overlap are applied. The field of view, i.e., the intersection of two dewarped perspectives, is $87 \mathrm{~mm} \times 40 \mathrm{~mm}$. The perspective view leads to the gradation of spatial resolution, which is $0.59 \mathrm{~mm} \pm 15 \%$ in the spanwise direction and $0.24 \mathrm{~mm} \pm 8 \%$ in the wall-normal direction. For the averaged flow field of 1250 image pairs, the expanded uncertainty in velocity components is $0.16 \% U_{\infty}$.

\section{MICRO-SCALE AND LARGE-SCALE VORTICAL STRUCTURES}

Our experimental study starts with a dye visualization of the flow over the riblet section and inside riblet valleys (micro-scale). The results are then correlated with the stereoscopic PIV data obtained in the cross-stream plane which reveal the large-scale vortical structures produced by the convergent-divergent riblets across the boundary layer. By synthesizing the findings from both the dye visualization and the PIV measurement, the flow topology of convergent-divergent riblets is proposed. 
(a)

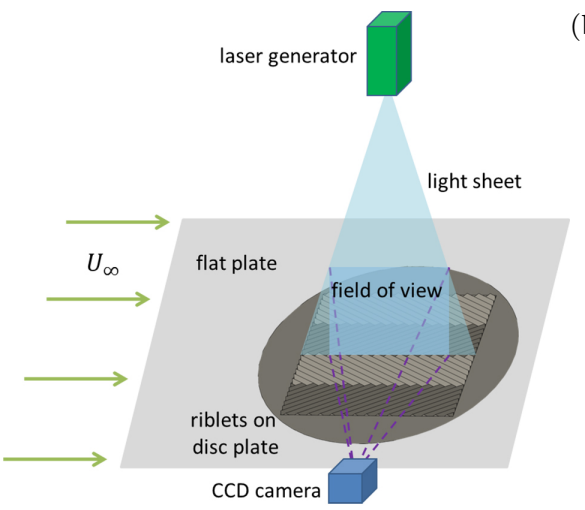

(b)

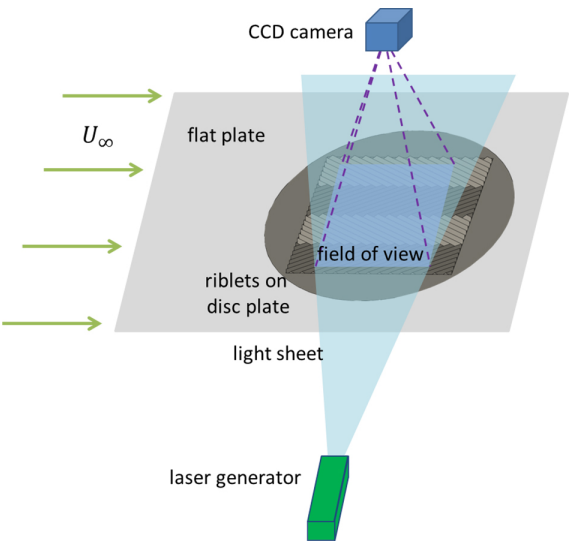

(c)

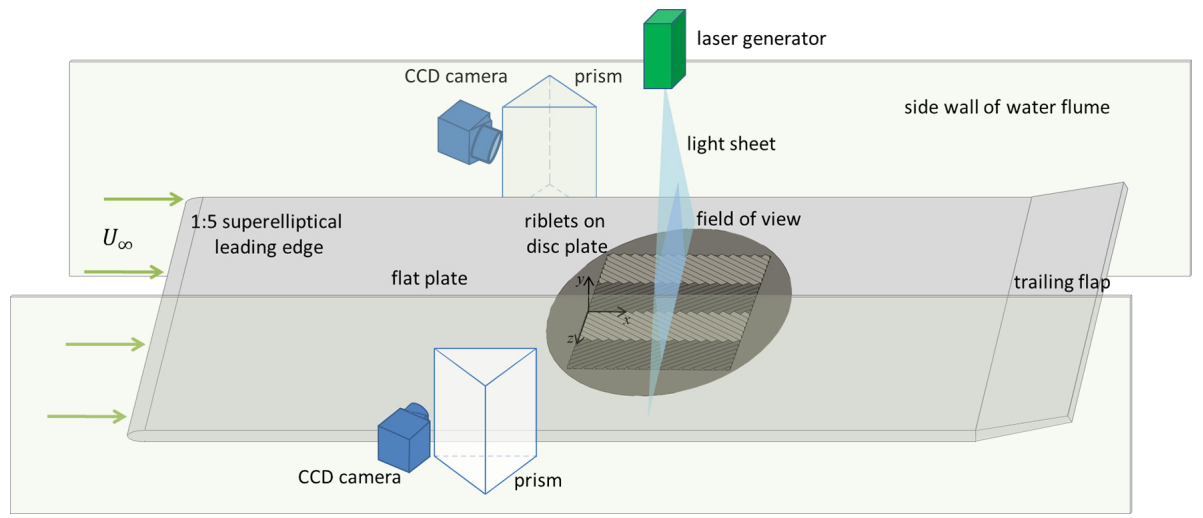

FIG. 3. Mono PIV system arrangement in (a) the longitudinal plane, (b) the wall-parallel plane. (c) Schematic diagram of the stereoscopic PIV system in the cross-stream plane. The test surface is inverted to enable a better illustration of the setup.

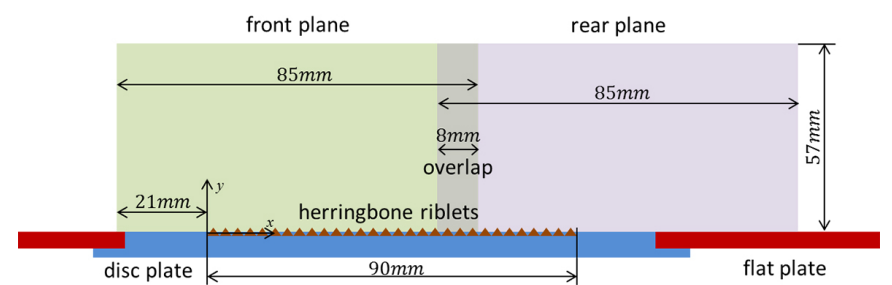

FIG. 4. Schematic of two measurement planes with an overlapping region in mono PIV measurements in the longitudinal plane.

\section{A. DYE VISUALIZATION OVER RIBLET SECTION}

The dye visualization experiment is carried out on convergent-divergent riblets with a wavelength of $45 \mathrm{~mm}$. Figure 5a shows the side view and the top view of the dye visualization images with the diverging line coinciding with the streamwise centerline of the disc plate. Red dye is injected through the central orifice $D$, which is located directly upstream of the diverging line. The images are instantaneous and they exhibit little changes in time indicating the steady nature of the flow. To illustrate the streaklines clearly, the side view is stretched vertically by a factor of 2.0 .
It can be seen from the dye images in the top view that, when the dye released from the orifice encounters the foremost apex of riblet ridges located along the diverging line, it splits into two streams $(S 3)$, one on each side of the diverging line. Each stream enters into a riblet valley and moves helicoidally along the valley till it reaches the converging line on the corresponding side. As the dye streamline reaches the converging line, it makes an abrupt turn and starts to flow in the streamwise direction. It is from this point $(P D 1)$ onwards that the dye starts to emerge from the riblet valley and to move above the riblet crest, making the streaklines becoming visible from the side view. As the dye flows through the riblet valley, a small portion of it spills over the riblet crest and is carried downstream by the main flow. The inner boundary of this overflow region over the riblet surface is indicated by the slant dashed lines. The overflow near the converging line is particularly strong producing further streaklines which are observed from the point $P D 2$ in the side view.

Dye visualization with the converging line coinciding with the streamwise centerline of the disc plate is also conducted by rotating the disc plate by $180^{\circ}$. This time, the dye is injected through an array of five orifices. The top view and the side view of the dye flow images are shown Fig. 5b. The symmetric distribution of streaklines 
in the top view confirms the high quality in setting up the experiment.

In the top view, the dye from orifice $C$ appears to follow the central converging line all the way downstream. The streakline from $M$ is seen to split into two streams $(S 1$ and $S 2)$ which enter the two adjacent riblet valleys respectively and flow towards the central converging line. Before arriving at the central converging line, a small portion of the dye $(S 2)$ spills over the riblet crest as overflow (gray dashed lines) and propagates downstream. The overflow of fluid above the riblet crest is unsteady, causing disturbances to the flow field. A majority of the dye reaches the converging line and becomes visible at $P M 2$ in the side view. Similar to what has already been observed in Fig. 5a, the dye streakline from orifice $D$ is seen to be split into two streams $(S 3$ and $S 4)$ when it encounters the foremost apex of riblet ridges. While $S 3$ follows one riblet valley and flows towards the central converging line, $S 4$ follows another riblet valley and flows towards another converging line located one wavelength apart at the edge of the riblet section. The slight asymmetric appearance in $S 3$ and $S 4$ seen in Fig. 5b is due to the ending of the riblet section on one side which is absent in Fig. 5a. To minimize the impact of the end effect on the results, the PIV data presented in this paper are always taken with the corresponding converging/diverging line as close to (if not on) the spanwise central line of the disc plate. In the top view, the overflow of the dye over the riblet crest lines as the dye flow through riblet valleys towards the converging lines can be observed clearly.

In the side view, one can see that the streakline originated from orifice $C$ emerges from the wall surface at $P C$ and it continues to move further away from the wall as it propagates downstream. Since the dye below the riblet crest is invisible in the side view, there exists a small offset between the streamwise locations where the dye is seen to enter the converging line in the top view and where it becomes visible in the side view. Over this distance, the dye moves along the converging line in the streamwise direction and lifts up in the wall-normal direction before reaching the crest level. Upon reaching the central converging line, the streakline $S 1$ lifts up vertically and becomes visible at $P M 1$. Since the two streaklines from $P M 1$ and $P M 2$ are very close in wall-normal position, they appear as one streakline in the side view. The streakline $S 3$ eventually appears in the side view at $P D 1$.

From the direction of movement of the dye $(S 1, S 2$, and $S 3$ ) in the top view, one can conclude that a secondary flow over the riblets from the diverging line towards the adjacent converging line is generated by convergent-divergent riblets. The flow gathers along the converging line and then lifts up away from the wall as revealed in the side view. Longitudinal riblets are known to stabilize the turbulent crossflow inside riblet valleys and to suppress near-wall momentum transfer in the spanwise direction. ${ }^{7,16,27}$ The convergent-divergent riblets seem to affect the crossflow differently.

\section{B. THE HELICOIDAL MOTION INSIDE RIBLET VALLEYS}

The dye visualization images in Figs. 5a and 5b clearly show that the dye injected from the five orifices does not travel inside a riblet valley in a straight line; it moves from side to side and also up and down. In fact, it moves along a helicoidal path inside the riblet valley, i.e., the fluid rotates around an axis as it moves along the riblet valley. The flow inside the riblet valley can be regarded as a channel flow under the influence of a freestream crossflow with a yaw angle $\gamma$. The interaction between the crossflow and the axial flow along the valley generates a secondary flow inside the valley and hence the helicoidal motion. A close-up view of the streaklines of dye, which is injected through four orifices $(H 1$ to $H 4)$ located at the bottom of riblet valleys next to the central diverging line, also confirms this. The (right-handed) helicoidal path of the dye is clearly revealed in Fig. 6a and depicted schematically in Fig. 6b. In the latter figure, the dashed line indicates the axis of the helix and the color gradient indicates the spatial location in the plane perpendicular to the axis. The sense of rotation of the helicoidal path follows the right-hand rule.

In Fig. 6a, it is also seen that the color intensity of streaklines varies significantly along the helicoidal path, and between the converging and diverging line a portion of the streaklines even temporarily disappears. This is caused by variations in the velocity along the helicoidal path. Within the riblet valley, the portion of the streakline close to the wall surface has a lower velocity and hence is less stretched. Consequently, it appears to be darker in color. In contrast, the portion of the streakline near the top of the valley travels faster, resulting in a reduction in the diameter of streakline and hence a lower dye concentration. As such, the color of the dye becomes lighter and the streakline may seem to have disappeared temporarily. The streakline becomes visible again further along the valley as its velocity reduces when it dives down to the bottom of the valley again. In Fig. 6a, the dashed lines indicate the locations when the streaklines originated from $H 1$ to $H 4$ touch the windward of each respective riblet valley. The pitch of the helicoidal motion is the distance along the valley covered by one complete rotation, which increases in the streamwise direction. An increase in pitch implies a higher axial component in the helicoidal motion.

\section{LARGE-SCALE VORTICAL STRUCTURES IN THE CROSS-STREAM PLANE}

The dye visualization images mainly provide information about the flow both inside riblet valleys and slightly above riblet crests. In order to examine the impact of convergent-divergent riblets on the entire boundary layer, stereoscopic PIV measurements are conducted. Figure 7 shows the in-plane velocity vectors at $x=12.5 \mathrm{~s} / \sin \gamma$ of 
(a)

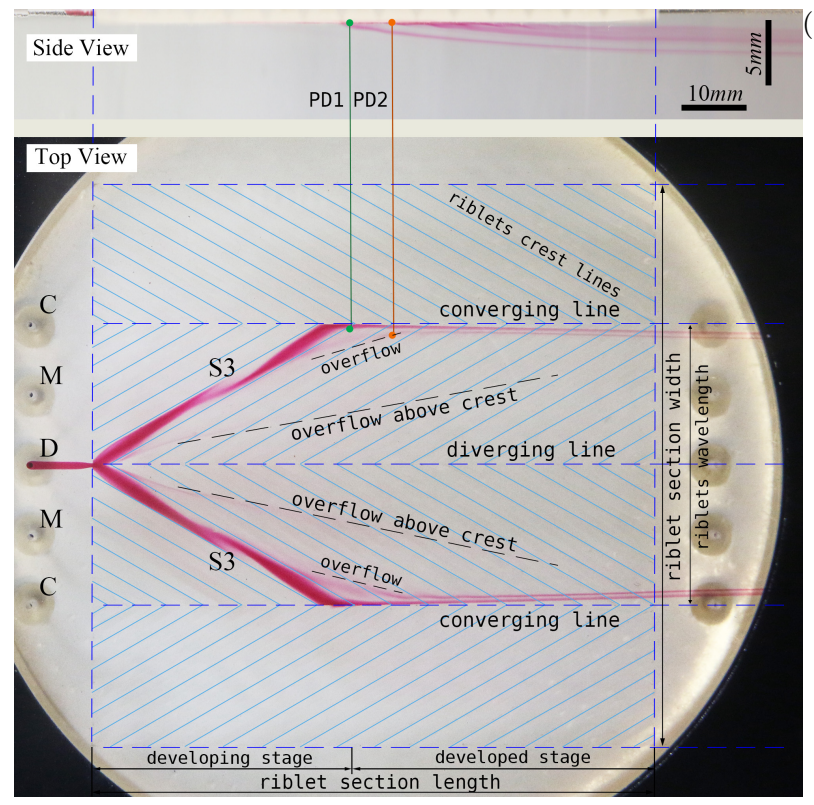

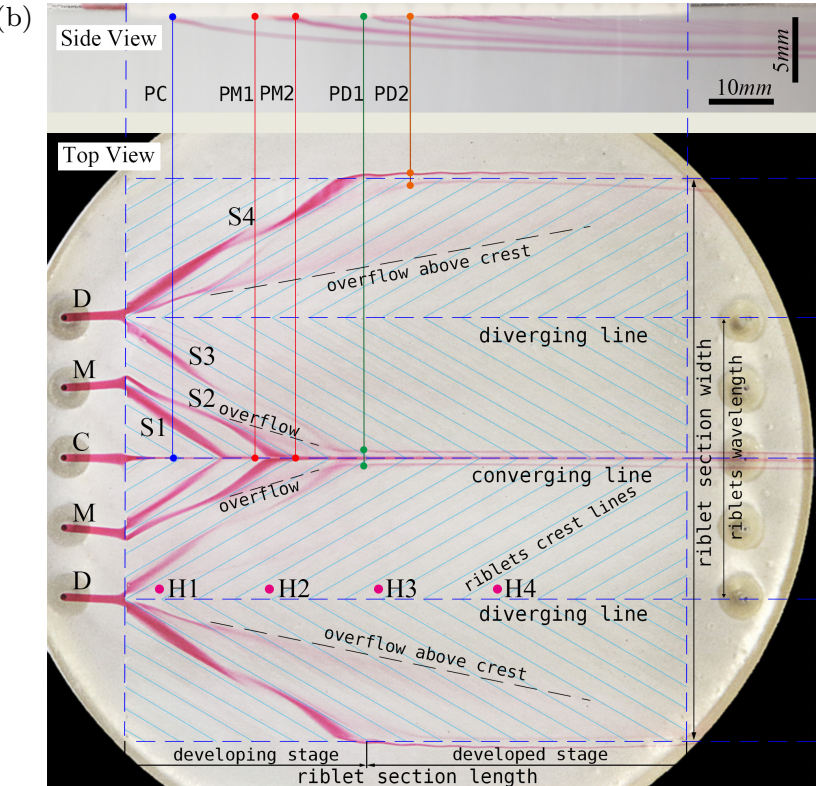

FIG. 5. Dye visualization in the side view (upper) and the top view (lower) with (a) the diverging line, (b) the diverging line coinciding with the streamwise centerline of the disc plate. Labels $C, D$ and $M$ indicate the dye orifices aligned with the converging line, the diverging line and the middle section in between respectively.

(a)

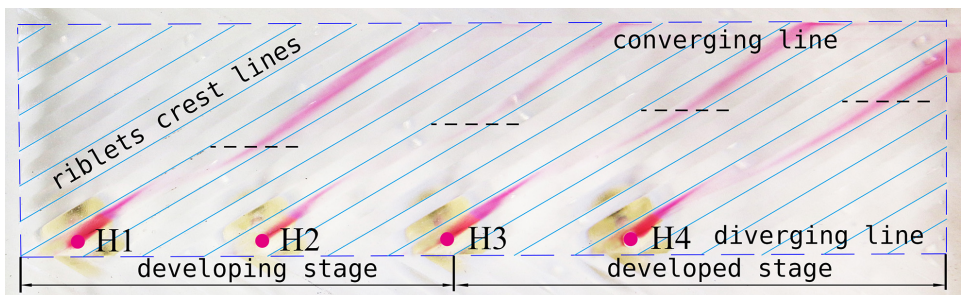

(b)

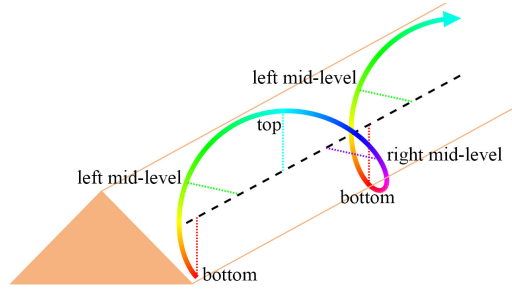

FIG. 6. (a) Dye visualization image of the helicoidal motion of the dye inside riblet valleys. Dye is injected to the bottom of the valleys through dye orifices $H 1$ to $H 4$ [refer to Fig. $5 \mathrm{~b}$ for locations]. (b) Schematic diagram of the helicoidal path of the dye inside a riblet valley in $3 D$ perspective.

convergent-divergent riblets with a wavelength of $30 \mathrm{~mm}$, $36 \mathrm{~mm}$ and $45 \mathrm{~mm}$ respectively. The contours of the signed swirling strength $\left\langle\lambda_{c i}\right\rangle$ and streamlines are also superimposed on the velocity vector field to assist the identification of vortex cores, if they are present. The signed swirling strength $\left\langle\lambda_{c i}\right\rangle=(\omega /|\omega|) \cdot \lambda_{c i}$ was proposed by Zhou et al. ${ }^{35}$ to analyze the local swirling motions, wherein $\lambda_{c i}$ is the imaginary part of the complex-conjugate eigenvalue of velocity gradient tensor and $\omega$ is the inplane vorticity. Here, $\left\langle\lambda_{c i}\right\rangle$ is calculated for each instantaneous flow field, and all the instantaneous snapshots (1250 image pairs in total) are then averaged to derive the contour in Fig. 7. Due to geometric symmetry, only the flow field over half of a wavelength of the convergentdivergent riblets is displayed. Triangles indicate the real geometries of the riblets. The dot/cross symbol indicates the center of the primary/secondary vortex. There are $4.3,5.2$ and 6.5 riblets respectively across the spanwise width of half of a wavelength for the three different riblet wavelengths studied here. Note that the horizontal axes of this figure are normalized with the riblet wavelength $(\Lambda)$, which is different among these three cases, and hence the flow structures are not scaled in physical size. In the spanwise direction, among every four velocity vectors only one vector is kept, and in the wall-normal direction every vector is plotted. The local boundary layer thickness of the flat plate is $\delta_{F P}=13.2 \mathrm{~mm}$.

In Fig. 7, a spanwise flow from the diverging line to the converging line in the near-wall region is clearly revealed by the direction of velocity vectors and this is consistent with that observed in the dye visualization images presented in Figs. 5a and 5b. It is also established that this near-wall flow is part of a large-scale recirculating secondary motion in the cross-stream plane which extend$\mathrm{s}$ itself across the entire boundary layer. In particular, an upwash motion dominates over the converging region whereas a downwash motion dominates over the diverging region. This observation is in agreement with the finding from previous experiments. ${ }^{23-25}$

A comparison of the control effects between 
convergent-divergent riblets and synthetic jet is provided. In the active flow control experiments with synthetic jets, the upwash and downwash of fluid in the near-wall region are induced by the rotations of hairpin legs, ${ }^{36,37}$ with an upwash leading to a decreased local skin friction and a downwash resulting in an increased one. For convergent-divergent riblets, the upwash and downwash in the near-wall region are produced by the highly directional spanwise surface patterns instead. Over the diverging line, the downward moving fluid is diverted along the valleys as it approaches the wall surface. The fluid subsequently collides over the converging region, leading to an increased skin friction drag whereas a decreased drag is obtained over the diverging line. ${ }^{24,25}$

A pair of co-rotating vortical structures with cores located at $y / \delta \approx 0.2$ can also be identified from the in-plane streamlines. For longitudinal riblets, such a large-scale vortical structure in the secondary flow is absent. ${ }^{16}$ Note the magnitude of the wall-normal or the spanwise component is about two orders of magnitude smaller than the freestream velocity, implying that the strength of these two vortical structures is quite weak. Furthermore, the signed swirling strength $\left\langle\lambda_{c i}\right\rangle$ has a high concentration at $y / \delta \approx 0.2$ near the diverging line whereas the magnitude of $\left\langle\lambda_{c i}\right\rangle$ is much lower near the converging line. Convergent-divergent riblets, with large-scale induced vortices in the secondary flow, may be applied to augment heat transfer. ${ }^{38-40}$

In order to investigate the reason behind the presence of the two co-rotating vortices revealed by the streamlines in Fig. 7, a zoom in view of the region near the diverging line is shown in Fig. 8. The contour of the vertical velocity and streamlines are superimposed on the velocity vector field to aid the analysis. The spanwise coordinate $z$ is normalized by the spanwise distance between adjacent riblets $\left(s_{z}=s / \cos \gamma=3 \mathrm{~mm} / \cos 30^{\circ}=3.46 \mathrm{~mm}\right)$.

It can been seen from Fig. 8 that in all the three cases the vortex near to the diverging line is associated with the presence of an upwash located below it and slightly to its right. Under the combined influence of this upwash motion and the spanwise crossflow, the local velocity vectors are pointing upward at an inclined angle. The reason behind the presence of this upwash motion can be explained as follows. The fluid inside the riblet valley is mainly supplied by the downwash over the diverging line. Due to its downward momentum, the fluid strikes upon the bottom of the valley and then deflected upwards. As such, some of the fluid will spill over the riblet crests, creating a local upwash at $z / s_{z} \approx 1.2$, as seen in Fig. 8. Here this upwash motion is termed "the overflow-induced upwash". It occurs at the windward of the riblet which intersects with the cross-stream PIV measurement plane. The vortex next to the diverging line (secondary vortex) is hence caused by the strong downwash over the diverging region and the overflow-induced upwash described above.

Immediately to the right of this upwash region along the span, there also exists a downwash region at $z / s_{z} \approx 2$ in which the velocity vectors are pointing downward at an inclined angle. This downwash motion is present as the result of the overflow-induced upwash, since a portion of the fluid from the crossflow needs to be entrained into the valley to satisfy the continuity requirement, and hence it is termed "the entrainment-induced downwash". In al1 these three cases, the location of the overflow-induced upwash is observed in the region around $1.0 s_{z}$, whereas the region of the entrainment-induced downwash lies around $2.0 \mathrm{~s}_{z}$. Their spanwise locations appear not affected by the riblet wavelength, suggesting that they are local to the downwash motion over the diverging line and the geometry of the riblets. The vortex close to the converging line (primary vortex) is caused by the strong upwash over the converging region and the entrainment-induced downwash described above.

It appears that as the riblet wavelength increases, the center of this vortex pair moves upward above $y / \delta_{F P}=$ 0.2 and the primary vortex becomes more dominant. In pervious experiments, ${ }^{24,25}$ only the primary vortex was observed and the secondary vortex close to the diverging region was not revealed. This could be attributed to the very large riblet wavelength $(\Lambda=147.5 \mathrm{~mm})$, more precisely the very large riblet wavelength to riblet spacing $(s=0.675 \mathrm{~mm})$ ratio, used in their experiments.

\section{FLOW TOPOLOGY AND FLOW MECHANISM}

In this section, the observations from the dye visualization and PIV results are synthesized to form the flow topology of convergent-divergent riblets. The schematic diagram of the flow topology is shown in Fig. 9a. Globally, it has established that a flow over the riblets from the diverging line towards the adjacent converging line is generated by convergent-divergent riblets [refer to Figs. 5 and 7]. This consequently leads to formation of a weak recirculating secondary flow across the entire boundary layer that creates a downwash motion over the diverging region and an upwash motion over the converging region [refer to Fig. 7]. The downwash over the diverging region provides feeding for the mass flow along the riblet valleys. Under the influence of the freestream crossflow above riblet crests, the fluid inside riblet valleys evolves along a helicoidal path [refer to the streaklines in Figs. 5 and 6]. Two co-rotating vortical structures are identified in the cross-stream plane within half of a wavelength for the riblet geometry studied in our experiment [refer to Fig. 7]. The secondary vortex close to the diverging region is generated by the downwash over the diverging line and the overflow-induced upwash at $z / s_{z} \approx 1$, whereas the primary vortex is caused by the upwash over the converging region and the entrainment-induced downwash at $z / s_{z} \approx 2$ [refer to Fig. 8]. As the riblet wavelength increases, the primary vortex becomes more dominant [refer to Fig. 7].

The flow topology over the surface of convergentdivergent riblets in Fig. 9a is better illustrated using $2 D$ sketches in the longitudinal planes at various representa- 
(a)

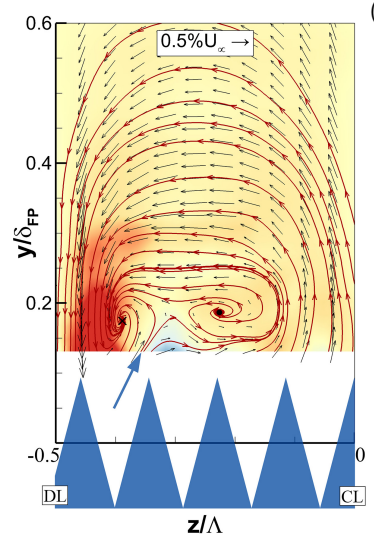

(b)

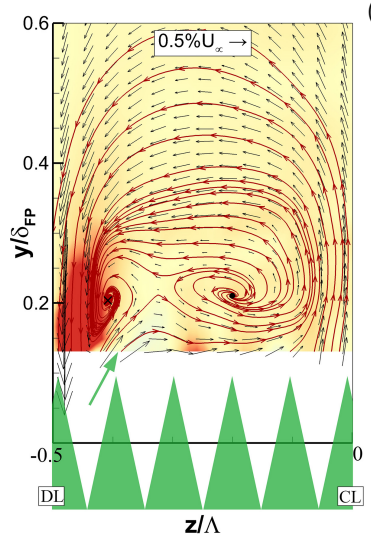

(c)

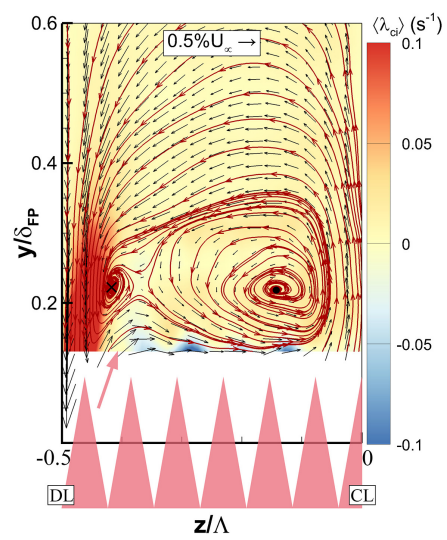

FIG. 7. Signed swirling strength contour, in-plane velocity vectors and streamlines of the time-averaged flow field of convergentdivergent riblets with wavelength (a) $\Lambda=30 \mathrm{~mm}$, (b) $\Lambda=36 \mathrm{~mm}$, (c) $\Lambda=45 \mathrm{~mm}$ at $x=12.5 \mathrm{~s}_{x}$. The region with counterclockwise swirling motion is red (positive), and the region with clockwise swirling motion is blue (negative).

(a)

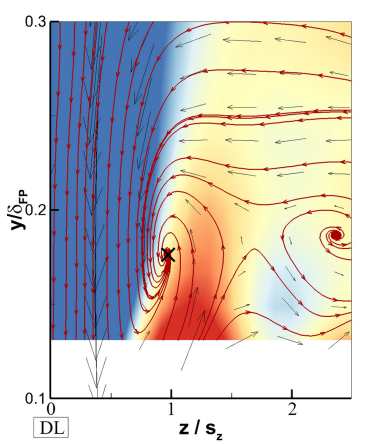

(b)

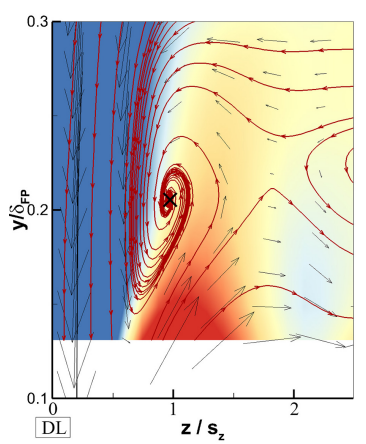

(c)

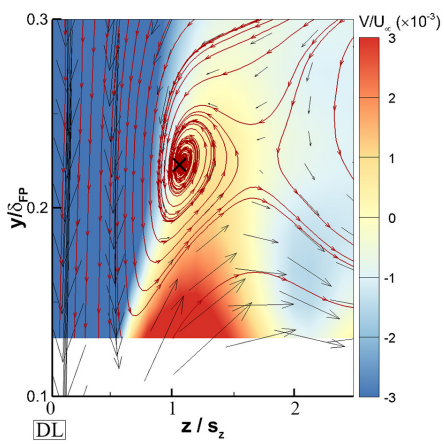

FIG. 8. Zoom-in view of the time-averaged flow field near the diverging line of convergent-divergent riblets with wavelength (a) $\Lambda=30 \mathrm{~mm}$, (b) $\Lambda=36 \mathrm{~mm}$, (c) $\Lambda=45 \mathrm{~mm}$ at $x=12.5 \mathrm{~s}_{x}$. The contour of vertical velocity and streamlines are superimposed on the velocity vector field. The cross symbol indicates the center of the secondary vortex near the diverging line.

tive spanwise stations as shown in Figs. 9b to 9g. In the longitudinal plane at $z=0$, the downwash dominates over the diverging line and under the influence of the freestream crossflow the local flow direction is pointing towards the windward side of the riblet valley, see Fig. 9b. As the fluid shunts along the riblet valley $\left(0<z / s_{z}<\right.$ $0.6)$, it is deflected upwards and towards the leeward side of the riblet valley, see Fig. 9c. In Fig. 9d, as the fluid impinges onto the leeward side and near the crest of the riblet valley $\left(0.7<z / s_{z}<1.7\right)$, a portion of it is taken away by the crossflow resulting in the overflow-induced upwash (overflow-1). As the fluid propagates along the riblet valley, some of the crossflow fluid is entrained into the riblet valley to compensate for the loss of fluid within the valley, leading to the entrainment-induced downwash, see Fig. 9e. Therefore, the entrainment-induced downwash $\left(1.8<z / s_{z}<2.2\right)$ is a direct consequence of the overflow-induced upwash. The overflow-induced upwash in Fig. 9d and the entrainment-induced downwash in Fig. 9e are the key contributing factors of the two co-rotating vortical structures seen in the cross-stream plane shown in Fig. 9a. As the fluid continues to propa- gate along the riblet valley, the freestream crossflow supplies the energy to the tangential velocity component of the helicoidal motion on the top of the riblet valley, see Fig. 9f. As the fluid on the top of the riblet valley is accelerated, a portion of the accelerated fluid moves above the riblet crest (overflow-2) from the windward side of the riblet valley. The overflow of fluid above the riblet crest is unsteady, causing disturbances to the flow field. In the longitudinal plane along the converging line $(z=\Lambda / 2)$, the upwash motion dominates, see Fig. 9g.

\section{E. DEVELOPING STAGE AND DEVELOPED STAGE}

Finally, the flow field over a riblet section of a finite length can be classified into two stages, i.e., the developing stage and the developed stage, depending on the main source of the fluid that sustains the spanwise flow over the riblets from the diverging line to the converging line. The developing stage is defined as the region from the start of the riblet section $(x=0)$ to a downstream station, where the upwash flow in the converg- 
(a)

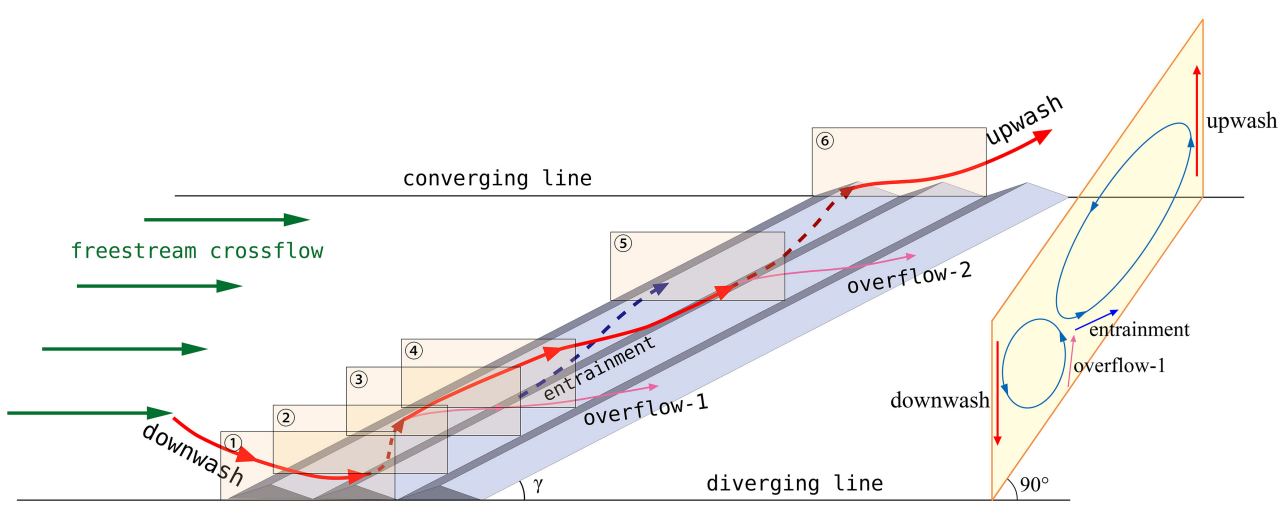

(b)

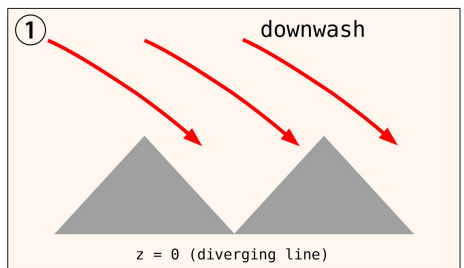

(e)

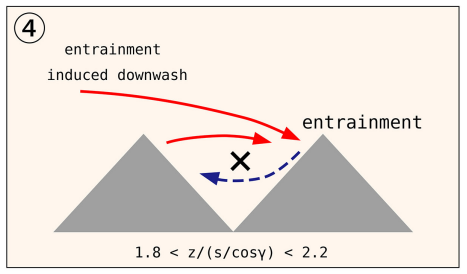

(c)

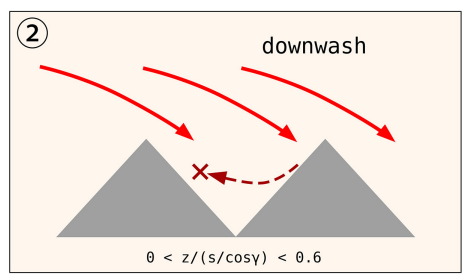

(f)

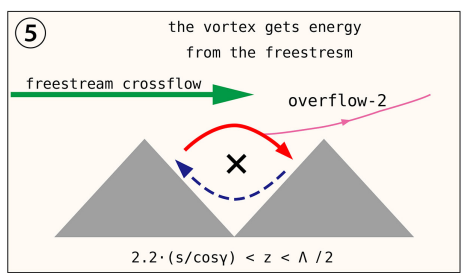

(d)

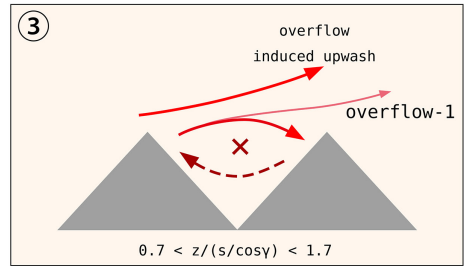

(g)

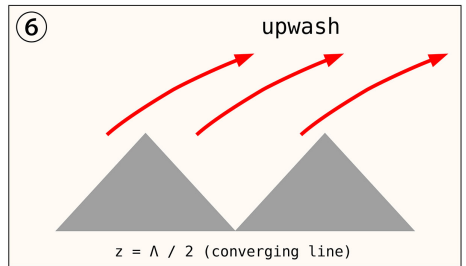

FIG. 9. Schematic diagram of the flow topology of $\Lambda=45 \mathrm{~mm}$ convergent-divergent riblets in (a) $3 D$ perspective, (b-g) multiple $2 D$ longitudinal planes.

ing region still comes from the crossflow boundary layer upstream of the riblet section. The developed stage is defined the region where the flow reaches the converging line mainly comes from the downwash motion over the diverging line. The approximate boundary between these two stages is located roughly at the location of $P D 1$ in Figs. 5a and 5b. Therefore, the length of the developing stage $(\Lambda /(2 \cdot \tan \gamma))$ depends on the riblet wavelength, with a longer wavelength resulting in a longer developing stage. The presence of the two stages has nothing to do with boundary layer transition. The difference in the flow characteristics in these two regions will be explored through the analysis of the PIV data obtained in longitudinal planes and a wall-parallel plane in the following sections.

\section{EFFECTS OF CONVERGENT-DIVERGENT RIBLETS ON THE DEVELOPMENT OF BOUNDARY LAYER}

In this section, the development of the boundary layer over convergent-divergent riblets is examined using PIV in the longitudinal plane and the wall-parallel plane. The measurement is made on the convergent-divergent riblets with a $\Lambda=45 \mathrm{~mm}$ wavelength. The characteristics of the induced velocity and vortical structures are aligned with the flow topology proposed in Fig. 9.

\section{A. FLOW FIELD IN THE LONGITUDINAL PLANE}

Firstly, the time-averaged velocity field in the longitudinal plane of the convergent-divergent riblets are examined and shown in Fig. 10. The vertical dashed line indicates the boundary of the developing stage and the developed stage. The $y$-coordinates are normalized by the local boundary layer thickness at $x=0\left(\delta_{F P}=12.7 \mathrm{~mm}\right)$ and the $x$-coordinates by the projected riblet spacing in $x$ direction $\left(s_{x}=s / \sin \gamma=6 \mathrm{~mm}\right)$.

The contours of the averaged streamwise velocity $U$ in the longitudinal planes over the converging line and the diverging line are shown in Figs. 10a and 10b respectively. PIV data very close to the riblet crest $\left(y / \delta_{F P} \leq 0.175\right)$ are not available due to the surface reflection of the laser light sheet, and this is indicated by the region void of con- 
tours close to the wall. In the longitudinal plane over the converging line (Fig. 10a), the region of low streamwise velocity (in blue) increases in height in the streamwise direction, indicating a reduction of the local velocity in the near-wall region. In contrast, over the diverging line (Fig. 10b), the region of low streamwise velocity decreases in height in the streamwise direction, indicating an increase of the local velocity near the wall. Due to the lack of experimental data very close to the wall in the present experiment, the wall shear stress is not presented in this paper.

The magnitude of the vertical velocity is much smaller than that of the streamwise velocity component. An intense upward motion of the fluid (upwash) is observed in the region over the converging line (Fig. 10c). The positive vertical velocity appears to be stronger over the leading edge of convergent-divergent riblets due to the blocking of convergent-divergent riblets. Furthermore, isolated vertical patches of positive vertical velocity are observed over the crests of convergent-divergent riblets. The upward motion appears to extend to the entire boundary layer with no clear physical upper boundary. For longitudinal riblets, the affected region is only limited to the vicinity of the riblet surface. ${ }^{26}$ In contrast, a profound downward motion (downwash) is seen in the area over the diverging line (Fig. 10d). The downwash appears to be confined within a finite region above the wall surface. It intensifies in the streamwise direction and decays abruptly once the riblet section ends. Over the diverging line, especially in the near-wall region, the magnitude of vertical velocity appears to increase in the streamwise direction indicating that the downwash motion is intensified further downstream. This is consistent with the observation of an increased helicoidal pitch along the streamwise direction as seen in Fig. 6a. Furthermore, isolated vertical patches of negative vertical velocity are observed over the valleys of convergent-divergent riblets. There is an expected strong correlation between the presence of an upwash/downwash and the reduction/increase of the streamwise velocity in the near-wall region.

Figures $10 \mathrm{e}$ and $10 \mathrm{f}$ show the contours of turbulence intensity $T u$, which is the velocity fluctuation $\left(\sqrt{u^{\prime 2}+v^{\prime 2}}\right)$ normalized by local in-plane velocity magnitude $\left(\sqrt{U^{2}+V^{2}}\right)$. Over the converging line, a region with elevated velocity fluctuations is observed, which is absent over the diverging line. As revealed by the top views of the dye visualization images in Figs. $5 \mathrm{a}$ and $5 \mathrm{~b}$, the overflows of the fluid above riblet crests generate disturbances over the riblet section. Due to the spanwise flow which directs from the diverging line to the converging line, such disturbances are brought towards the converging region, causing a substantial increase in the turbulence intensity there.

Contours of the time-averaged vorticity $\omega_{z}$ indicating shear and rotation in the longitudinal plane of convergent-divergent riblets are shown in Figs. 11a and 11b. The reversed colorbar is applied to highlight the high vorticity region. The level of vorticity in the bound- ary layer upstream of the riblet section $(x<0)$ can be considered as the baseline level. From Fig. 11a, one can see that over the converging line the level of vorticity is significantly enhanced and the region with high vorticity is displaced upward becoming detached from the wall in the developed region. The uplift of vorticity $\omega_{z}$ seen in Fig. 11a is a result of the upwash motion occurring over the converging region [refer to the side views in Figs. 5a and 5b]. On the contrary, over the diverging line weakened vorticity and downward movement of the high vorticity region are observed in accordance with the presence of a downwash motion. Based on the results presented in Figs. 11a and 11b, one can conclude that the fluid mixing is enhanced in the converging region and suppressed in the diverging region. Further analysis reveals that the enhancement or weakening of the shear mainly comes from the streamwise part of the fluid velocity rather than the vertical component.

In the vorticity $\omega_{z}$ shown in Figs. $11 \mathrm{a}$ and $11 \mathrm{~b}$, the contribution from the shear to the averaged velocity field dominates. In order to isolate the local swirling motion from the shear, the contours of signed swirling strength $\left\langle\lambda_{c i}\right\rangle=\left(\omega_{z} /\left|\omega_{z}\right|\right) \cdot \lambda_{c i}$ are also examined. $\left\langle\lambda_{c i}\right\rangle$ is calculated firstly for each instantaneous flow field, and all the instantaneous snapshots (1250 image pairs in total) are then averaged to produce the contours shown in Figs. 11c and 11d. Similar spatial periodicity in $\left\langle\lambda_{c i}\right\rangle$ can be obviously seen over the converging and diverging line. More intense vortical structures are observed over the diverging line than the converging line, which is consistent with the fact that the helicoidal motions within the riblet valleys are firstly generated over the diverging region and hence have a higher swirling strength.

\section{B. FLOW FIELD IN THE WALL-PARALLEL PLANE}

In the present experiment, in order to investigate the effects of convergent-divergent riblets on the secondary flow in the near-wall region, the PIV measurement is made in a wall-parallel plane very close to the wall surface. The exact location of the center of the laser sheet used in this measurement cannot be measured easily in practice. However, it is possible to estimate its distance to the wall from the streamwise velocity on this plane $(0.035 \mathrm{~m} / \mathrm{s})$ by assuming that the local velocity profile in the baseline boundary layer follows the Blasius profile. From this, the wall-parallel plane is estimated to be located at $3 \mathrm{~mm}$ above the smooth wall surface or $1.8 \mathrm{~mm}$ above the crest of convergent-divergent riblets.

Due to the longitudinal growth of the laminar boundary layer, the streamwise velocity in this wall-parallel plane decreases in the streamwise direction. The effec$\mathrm{t}$ of streamwise variation can be removed by subtracting the streamwise velocity $U_{0}$ obtained in the boundary layer over the smooth wall from that measured over the convergent-divergent riblets. In Figs. $12 \mathrm{a}$ and $12 \mathrm{~b}$, the contours of the induced streamwise velocity $(\Delta U=$ 
(a)

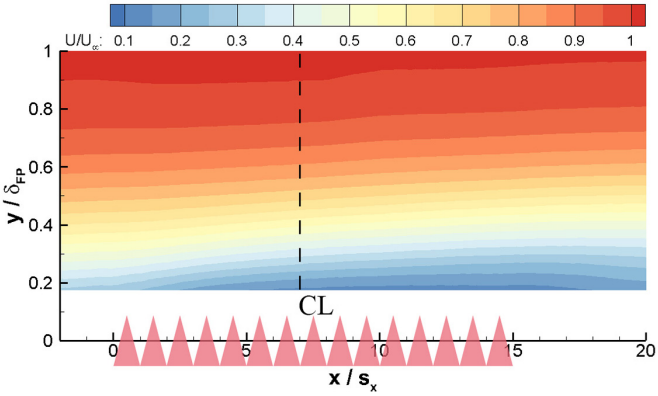

(c)

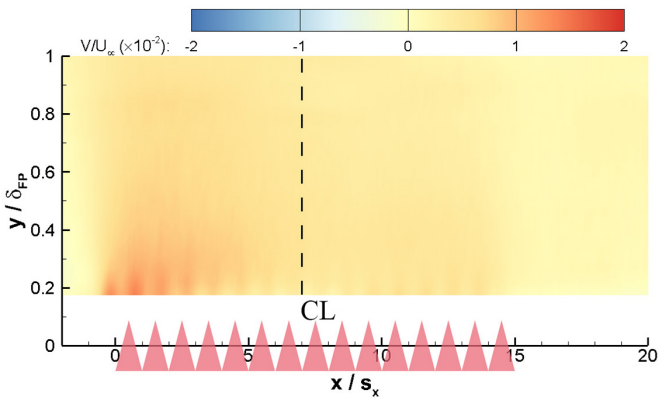

(e)

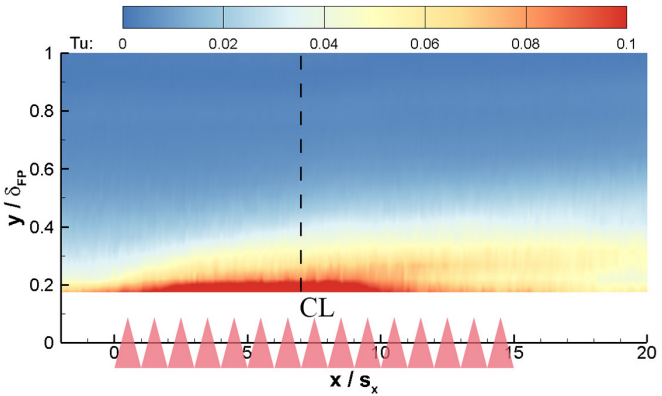

(b)

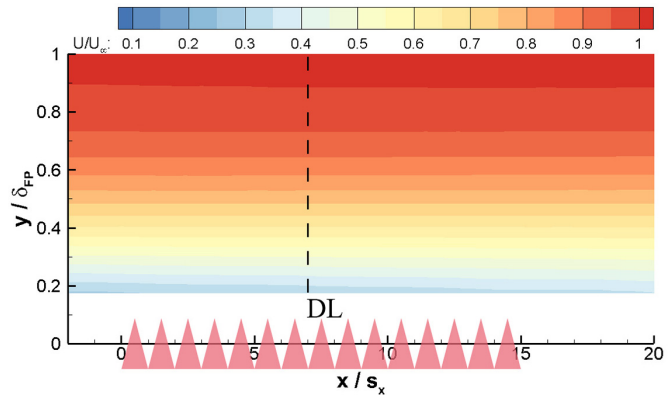

(d)

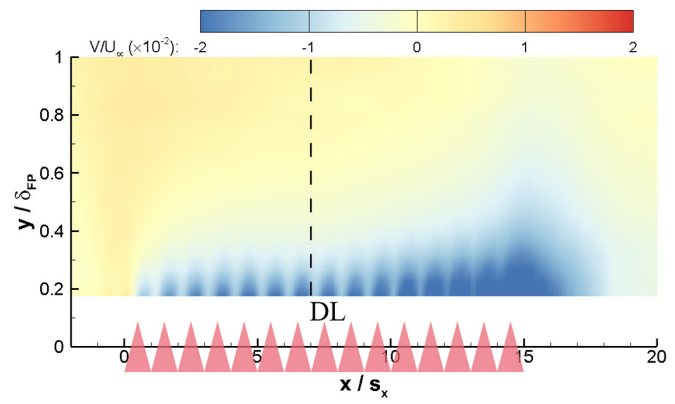

(f)

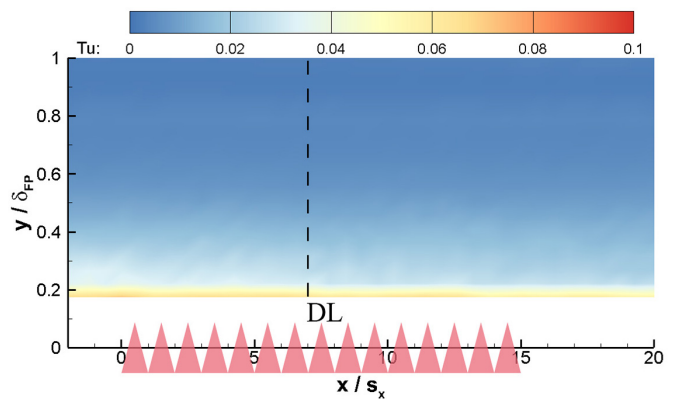

FIG. 10. Contour of (a,b) time-averaged streamwise velocity $U,(\mathrm{c}, \mathrm{d})$ time-averaged vertical velocity $V$, (e,f) turbulence intensity $T u$ of $\Lambda=45 \mathrm{~mm}$ riblets in the longitudinal plane over the converging line (left) and the diverging line (right).

$\left.U-U_{0}\right)$ reveal the presence of concentrated zones of streamwise velocity deficit/excess in stripy shape over the converging/diverging regions. The vertical dashed line indicates the boundary between the developing stage and the developed stage, and the horizontal dash-dotdot lines indicate the overflow-induced upwash. Channeling of the flow with low-speed and high-speed pathways was also observed in the boundary layer over turbine blades covered with particle deposits ${ }^{41}$ or convergentdivergent riblets. ${ }^{32}$ The appearance of concentrated zones of streamwise velocity deficit/excess implies that the upwash/downwash motions occur predominantly over the converging/diverging region. They persist downstream of the riblet section $\left(x>15 s_{x}\right)$. It is also noticed that a slightly negative velocity occurs in the remaining region, indicating that the whole flow field is affected by the riblet section. The spanwise position of the overflow-induced upwash near the diverging region does not change in the developed stage, which is indicated by the green lines.

To illustrate the streamwise development, spanwise distributions of the induced streamwise velocity at dif- ferent streamwise stations are shown in Figs. 12c and 12d. Over the converging line, both the magnitude and the width of velocity deficit increase progressively till the end of the riblet section. Over the diverging region, both the magnitude and the width of velocity excess increase progressively and such an increase continues even after the end of the riblet section. It is noticed that the zone of velocity deficit is broader and has a higher maximum magnitude than that of the zone of velocity excess. A localised decrease in the streamwise velocity is also observed near the boundary of the velocity excess zone, which is indicated by the dotted circles in Fig. 12d and associated with the overflow-induced upwash. The local upwash is also observed in the cross-stream plane, as indicated by the arrow in Fig. 7c and the contour of vertical velocity in Fig. 8c.

In Fig. 13, the contour of vorticity $\omega_{y}$ and signed swirling strength $\left\langle\lambda_{c i}\right\rangle$ in the wall-parallel plane are shown. The vorticity in Fig. 13a represents both shear and rotation of the averaged velocity field, whereas the signed swirling strength in Fig. 13b indicates only the swirling motions. In Fig. 13a, longitudinal pairs of high 
(a)

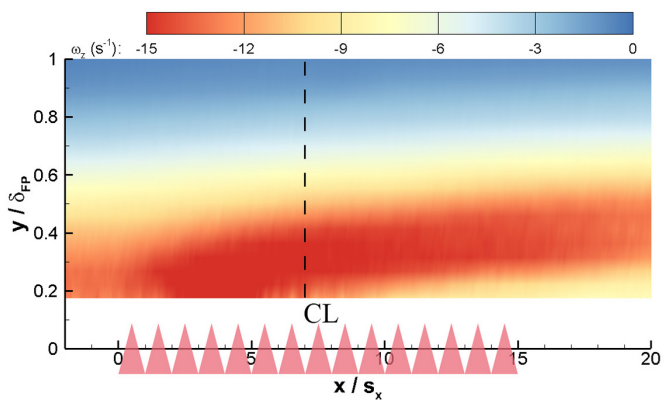

(c)

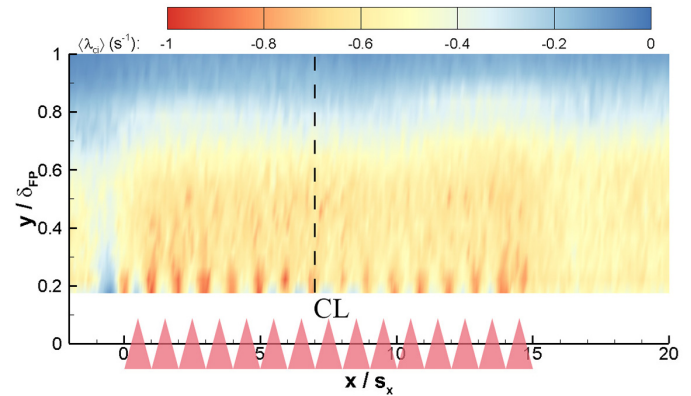

(b)

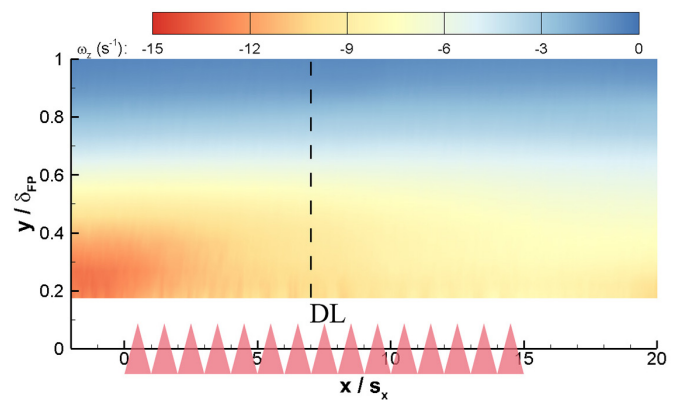

(d)

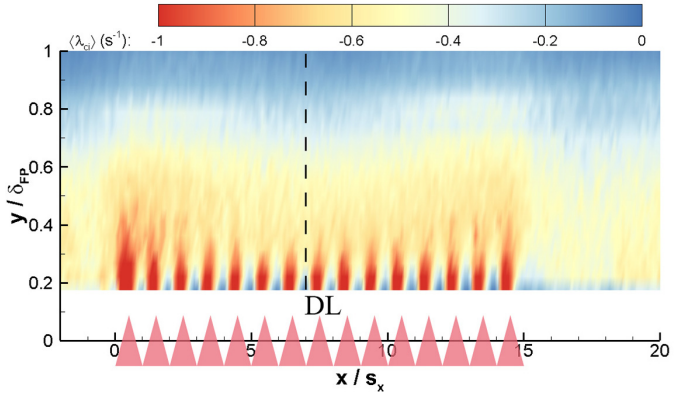

FIG. 11. Contour of $(\mathrm{a}, \mathrm{b})$ the time-averaged vorticity $\omega_{z},(\mathrm{c}, \mathrm{d})$ the signed swirling strength $\left\langle\lambda_{c i}\right\rangle$ of $\Lambda=45 \mathrm{~mm}$ riblets in the longitudinal plane over the converging line (left) and the diverging line (right). The region with counterclockwise rotational/swirling motion is blue (positive), and the region with clockwise motion is red (negative).

(a)

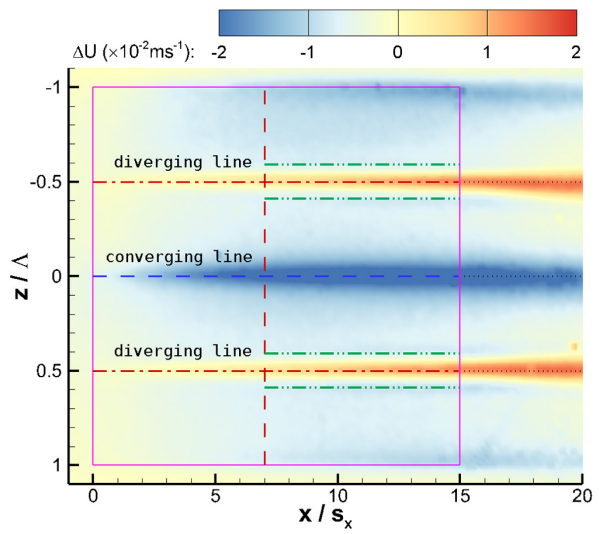

(c)

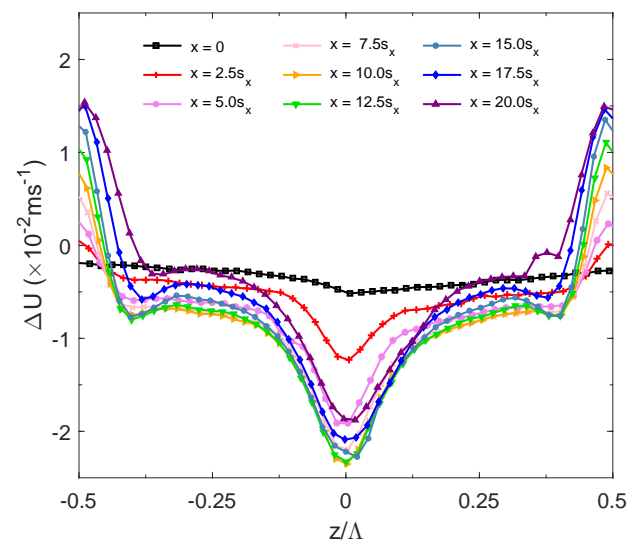

(b)

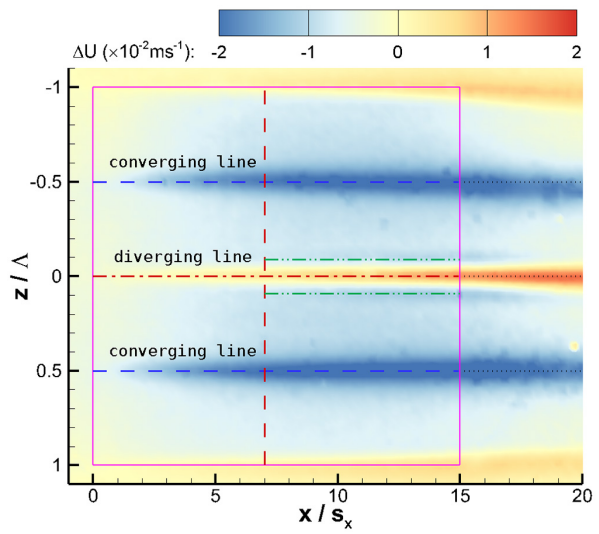

(d)

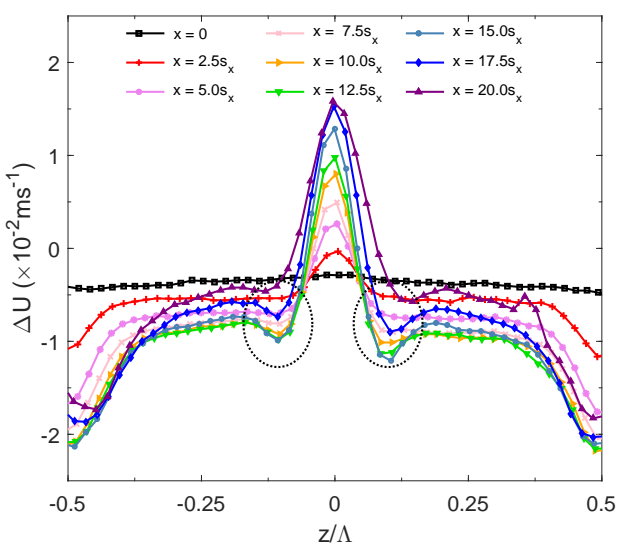

FIG. 12. Contour and profile of the time-averaged induced streamwise velocity $\Delta U=U-U_{0}$ of $\Lambda=45 m m$ riblets in the wall-parallel plane with $(\mathrm{a}, \mathrm{c})$ the converging line at $z=0,(\mathrm{~b}, \mathrm{~d})$ the diverging line at $z=0$. 
negative and positive vorticity straddle along the converging line and the diverging line due to the large local velocity gradient across these lines, see the bell-shaped profiles of the induced streamwise velocity in Figs. 12c and $12 \mathrm{~d}$. Between the converging line and the diverging line, shear motions are very weak and the magnitude of vorticity is close to zero.

In Fig. 13b, longitudinal pairs of high negative and positive swirling strength are seen to straddle along the converging/diverging line in a similar manner as in Fig. 13a. However, with a closer examination of the zoomed in regions, one can see that in the developing stage in Fig. 13c the intensity of $\left\langle\lambda_{c i}\right\rangle$ is low and appears to be featureless, whereas in the developed stage in Fig. 13d the intensity of $\left\langle\lambda_{c i}\right\rangle$ increases to such an extent that isolated patches within each riblet valley with intense swirling strength appear. The regions with intense swirl are located between two adjacent crest lines, which can be interpreted as the imprints of the vortical structures inside riblet valleys on the wall-parallel plane. The swirling motions are more intense near the diverging line since vortical structures inside riblet valleys are initially generated there. It is observed that although the strength of vorticity is maintained, the swirling motion decays more rapidly once the riblet section ends.

\section{EFFECTS OF RIBLET WAVELENGTH}

In this section, the effects of riblet wavelength on the flow field characteristics, including induced streamwise velocity, vorticity and swirling, are investigated using PIV measurements.

\section{A. PARAMETERS OF INDUCED VELOCITY AND VORTICITY}

In order to enable a quantitative comparison of the amplitude and width of the upwash/downwash motion occurring over the converging/diverging region of riblets with different wavelengths, a parameter extraction algorithm for the bell-shaped velocity profiles obtained in the wall-parallel plane is developed. In Fig. 14a, the $C^{2}$ continuity spline interpolation (curve) is applied to provide a smooth curve fit of the discrete data points (delta symbols). The base value is defined as the averaged value in the plateau region between converging line and diverging line, and the amplitude $\left(A_{\Delta U}\right)$ represents the difference between the extreme value and the base value. The width of the upwash/downwash region over the converging/diverging line $\left(W_{\Delta U}\right)$ is defined as the width of the curve with a $10 \%$ amplitude above the base value.

Figure 14b shows the variations of the amplitude of the induced streamwise velocity $\left(A_{\Delta U}\right)$ along the converging and diverging line for convergent-divergent riblets with three different wavelengths. Along the converging line, the amplitude firstly increases continuously, and it then levels off after reaching the peak value. In contrast, along the diverging line the amplitude exhibits a continuous increase towards the end of the riblet section before it decreases slightly afterwards. This results in a stronger downwash over the diverging line than the upwash over the converging line towards the end of the riblet section. Interestingly, it is also found that the point where the amplitude begins to level off along the converging line approximately coincides with the beginning of the developed stage. Furthermore, it is observed that an increase in riblet wavelength leads to a larger amplitude of the induced streamwise velocity over both the converging and the diverging regions.

Figure 14c shows the variations of the width of the induced streamwise velocity $\left(W_{\Delta U}\right)$ along the converging and diverging line for convergent-divergent riblets with three different wavelengths. For all riblet wavelengths, the region with upwash centered at the converging line is substantially wider than the region with downwash straddling along the diverging line. The overflow near the converging line enhances the upwash, whereas the overflow close to the diverging region weakens the downwash. Downstream of the riblet section $\left(x>15 s_{x}\right)$, the width of the downwash exhibits an increasing trend, whereas the width of the upwash remains basically constant. In the current wall-parallel plane, a larger wavelength leads to wider region with upwash centered at the converging line. An opposite trend is observed for the region with downwash over the diverging line, i.e., a larger wavelength leads to a narrower region.

Figure 15 compares the magnitude of vorticity $A_{\omega_{y}}$, which is the mean magnitude of the positive and negative peaks in the spanwise vorticity profiles in the wallparallel plane. Along the converging line, $A_{\omega_{y}}$ exhibits a continuous increase in the developing stage and then levels off in the developed stage. Along the diverging line, $A_{\omega_{y}}$ increases up to the end of the riblet section $\left(0<x<15 s_{x}\right)$ and it decreases rapidly further downstream $\left(x>15 s_{x}\right)$. A higher $A_{\omega_{y}}$ close to the diverging line indicates more intense shear and rotation within that region. The wavelength of riblets has a negligible influence on $A_{\omega_{y}}$ over the converging region. On the other hand, a larger wavelength leads to a higher $A_{\omega_{y}}$ in the region over the diverging region. Over the diverging region, since the amplitude of the induced velocity is larger and the width in the diverging region is narrower [refer to Figs. 14b and 14c], the shear and rotation are more intense than those near the converging line. In additional, it is also found that changes in riblet wavelength have a negligible influence on the spanwise width of vorticity peaks along both the converging and the diverging lines (the graph is not shown here for brevity).

The main contributor to the time-averaged vorticity $\left(\omega_{y}=\partial U / \partial z-\partial W / \partial x\right)$ in Fig. 13a is the spanwise gradient of the streamwise velocity, i.e., $\partial U / \partial z$. The effects of riblet wavelength on the amplitude and width of the induced streamwise velocity $\Delta U$ have been revealed in Figs. 14b and 14c respectively. Along the diverging line, 
(a)

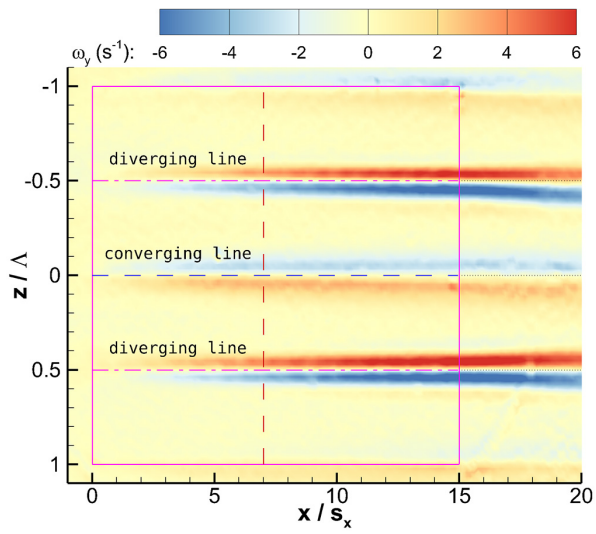

(c)

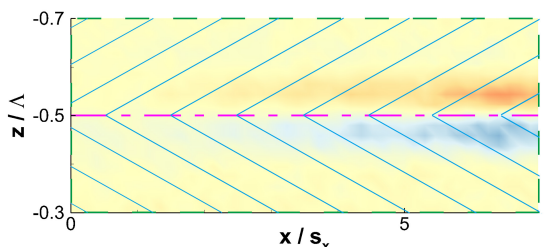

(b)

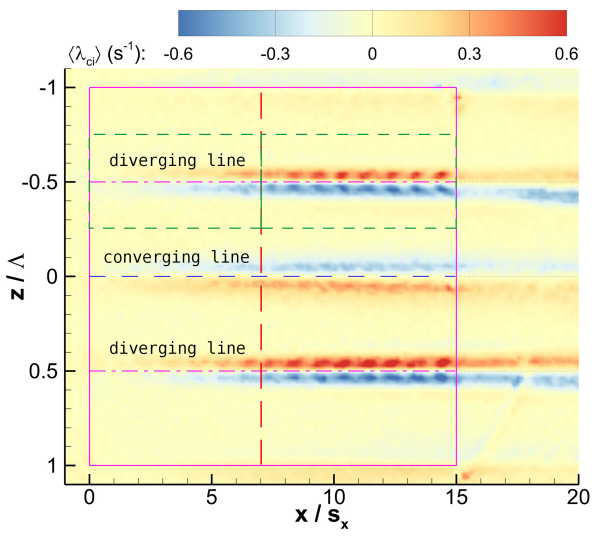

(d)

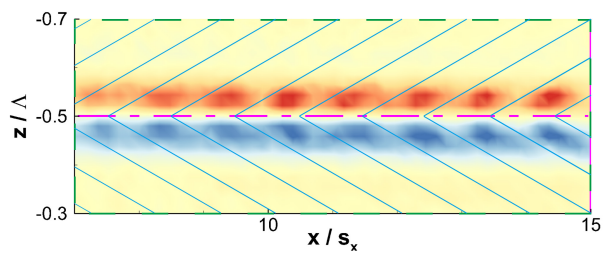

FIG. 13. Contour of (a) the time-averaged vorticity $\omega_{z}$ and (b) the signed swirling strength $\left\langle\lambda_{c i}\right\rangle$, zoomed in region in (c) the developing stage and (d) the developed stage in the wall-parallel plane of $\Lambda=45 \mathrm{~mm}$ riblets with the converging line at $z=0$. The region with counterclockwise rotational/swirling motions is red (positive), and the region with clockwise motions is blue (negative).

(a)

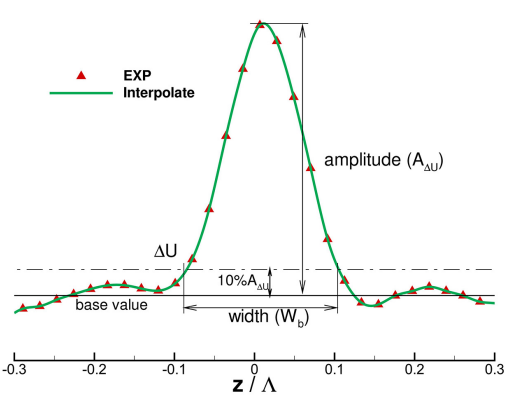

(b)

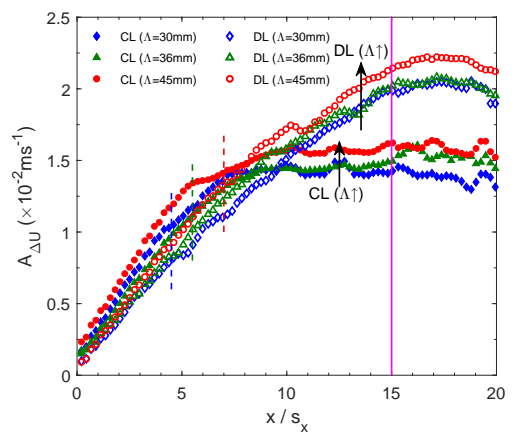

(c)

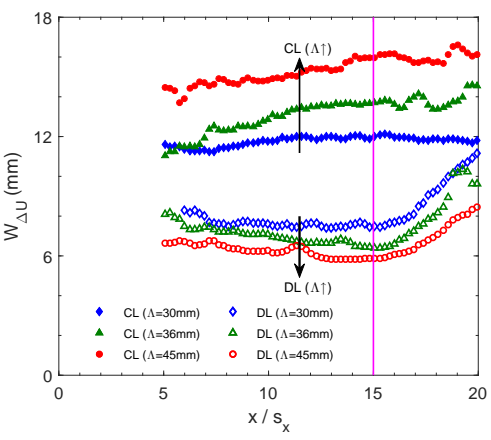

FIG. 14. (a) Schematic of the definition of parameters, (b) amplitude $A_{\Delta U}$ and (c) width $W_{\Delta U}$ of the upwash region and the downwash region in the wall-parallel plane for convergent-divergent riblets with three different wavelengths.

the amplitude $A_{\Delta U}$ increases whereas the width $W_{\Delta U}$ decreases as the riblet wavelength increases, leading to a dramatic increase in $\partial U / \partial z$. On the contrary, along the converging line, both the amplitude $A_{\Delta U}$ and the width $W_{\Delta U}$ increase as the riblet wavelength increases. Thus, an increasing riblet wavelength is expected to have a negligible effect on $A_{\omega_{y}}$ in the converging region.

\section{B. THE DECELERATION EFFECT}

In Fig. 12, contours of the induced streamwise velocity in the wall-parallel PIV measurement plane reveal changes in streamwise velocity introduced by the riblets with reference to that by a smooth wall. In order to quan- tify the effect of convergent-divergent riblets on the flow at each streamwise station in this plane, we introduce a new parameter, which is the integral of the induced streamwise velocity across one complete wavelength at the corresponding station, i.e., $T I U=\int_{-\Lambda / 2}^{+\Lambda / 2} \Delta U(z) d z$. The absolute value of $T I U$ indicates the degree by which the flow is accelerated or decelerated by the convergentdivergent riblets. A positive $T I U$ implies an acceleration effect whereas a negative value implies a deceleration effect across the span.

Based on the results shown in Fig. 16, convergentdivergent riblets are found to generate a deceleration, with a larger riblet wavelength resulting in a more intense deceleration. The value of TIU decreases rapidly in the developing stage as the deceleration effect enhances. In 


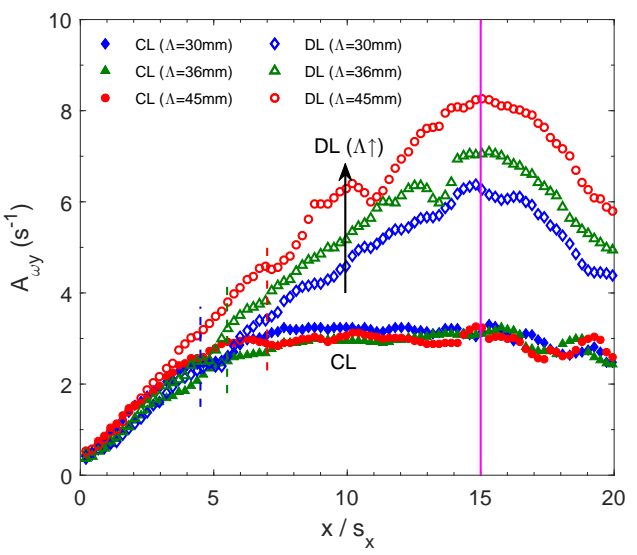

FIG. 15. Comparison of the magnitude of vorticity $A_{\omega_{y}}$ in the wall-parallel plane for convergent-divergent riblets with three different wavelengths.

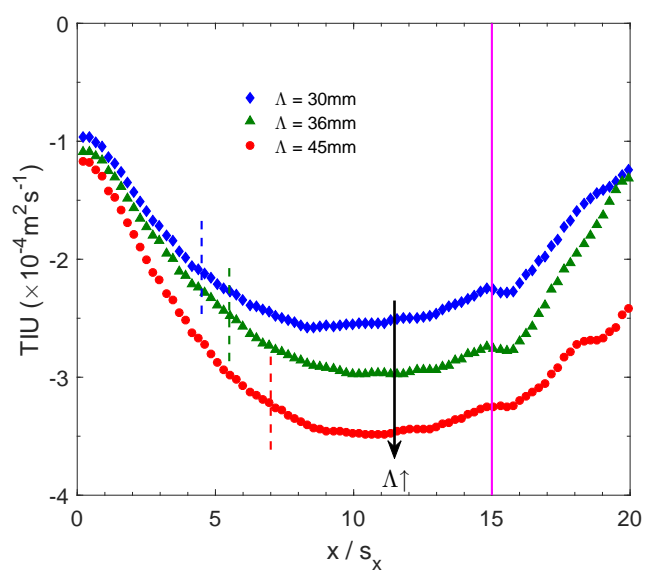

FIG. 16. Streamwise variations in $T I U$ for convergentdivergent riblets with three different wavelengths. The boundaries between the developing stage and the develope$\mathrm{d}$ stage are indicated by the dashed vertical lines.

the developed stage, the value of $T I U$ first decreases slowly, and then increases slightly. Once the riblet section ends $\left(x>15 s_{x}\right)$, the deceleration effect begins to weaken rapidly.

\section{CONCLUSION}

In this work, the development of a laminar boundary layer over a rectangular convergent-divergent riblet section with a finite streamwise length is studied experimentally using dye visualization and PIV at a freestream velocity of $0.1 \mathrm{~m} / \mathrm{s}$ in a water flume. The flow topology over this highly directional spanwise roughness is established and the impact of riblet wavelength on vortical structures is also revealed for the first time. The key findings from this study are summarized as follows:

Firstly, it has established that convergent-divergent ri-

\begin{tabular}{ccc}
\hline \hline Properties & Converging Region & Diverging Region \\
\hline$A_{\Delta U}$ & $\uparrow$ & $\uparrow$ \\
$W_{\Delta U}$ & $\uparrow$ & $\downarrow$ \\
$A_{\omega_{y}}$ & $\rightarrow$ & $\uparrow$ \\
\hline \hline
\end{tabular}

TABLE II. Variations of the flow field properties with increased wavelength of convergent-divergent riblets. The symbol $\uparrow$ indicates increase, $\downarrow$ denotes decrease, and $\rightarrow$ represents no significant effect.

blets generate a flow close to the riblets from the diverging line towards the adjacent converging line. This consequently leads to the formation of a weak recirculating secondary flow in cross-stream plane across the entire boundary layer that creates a downwash motion over the diverging region and an upwash motion over the converging region. The downwash over the diverging region provides a feeding mechanism for the mass flow along riblet valleys. Under the influence of the crossflow above riblet crests, the fluid inside the riblet valley evolves along a helicoidal path. It also interacts with the crossflow boundary layer and hence plays a key role in determining the structure of the secondary flow across the boundary layer.

Secondly, in consistent with the findings from other researches, a reduction in the streamwise velocity and an increase in turbulence and vorticity is found in the longitudinal plane along the converging line and the opposite is observed in the longitudinal plane along the diverging line. Furthermore, the streamwise development of the flow over the riblet section can be divided into a developing stage followed by a developed stage. In the developing stage, the magnitudes of induced streamwise velocity and vorticity on the converging line continue to increase whereas in the developed stage the values of these parameters remain essentially unchanged.

Finally, it is found that a larger wavelength produces a higher magnitude of upwash/downwash in the converging/diverging region and hence leads to a more intense secondary flow. A more intense deceleration effect is also observed for riblets with larger wavelength. The effects of riblet wavelength on the parameters of the flow field are summarized in Table II.

\section{ACKNOWLEDGMENTS}

The authors would like to thank the workshop technicians at School of Mechanical, Aerospace and Civil Engineering for their excellent technical supports during the setting up of our experimental rig. They also would like to acknowledge the timely technical advice provided by Dr. Amine Koched and Dr. Martin Hyde at TSI. Finally, the authors wish to thank the referees of this paper for their valuable review comments which have enabled us to make a substantial improvement to this paper. 
${ }^{1} \mathrm{M}$. Walsh, "Turbulent boundary layer drag reduction using riblets," in 20th Aerospace Sciences Meeting (American Institute of Aeronautics and Astronautics, Reston, Virigina, 1982).

${ }^{2} \mathrm{M}$. Walsh and A. Lindemann, "Optimization and application of riblets for turbulent drag reduction," in 22nd Aerospace Sciences Meeting (American Institute of Aeronautics and Astronautics, Reston, Virigina, 1984).

${ }^{3}$ H. Choi, P. Moin, and J. Kim, "On the effect of riblets in fully developed laminar channel flows," Physics of Fluids A: Fluid Dynamics 3, 1892-1896 (1991).

${ }^{4}$ L. Djenidi, F. Anselmet, J. Liandrat, and L. Fulachier, "Laminar boundary layer over riblets," Physics of Fluids 6, 2993-2999 (1994).

${ }^{5}$ S. Raayai-Ardakani and G. H. McKinley, "Drag reduction using wrinkled surfaces in high Reynolds number laminar boundary layer flows," Physics of Fluids 29, 093605 (2017).

${ }^{6}$ G. R. Grek, V. V. Kozlov, S. V. Titarenko, and B. G. Klingmann, "The influence of riblets on a boundary layer with embedded streamwise vortices," Physics of Fluids 7, 2504-2506 (1995).

${ }^{7}$ K.-S. Choi, "Near-wall structure of a turbulent boundary layer with riblets," Journal of fluid mechanics 208, 417-458 (1989).

${ }^{8}$ A. G. Kravchenko, H. Choi, and P. Moin, "On the relation of nearwall streamwise vortices to wall skin friction in turbulent boundary layers," Physics of Fluids A: Fluid Dynamics 5, 33073309 (1993).

${ }^{9}$ D. W. Bechert, M. Bruse, W. Hage, J. G. T. Van Der Hoeven, and G. Hoppe, "Experiments on drag-reducing surfaces and their optimization with an adjustable geometry," Journal of Fluid Mechanics 338, 59-87 (1997).

${ }^{10}$ M. j. Walsh, "Riblets as a Viscous Drag Reduction Technique," AIAA Journal 21, 485-486 (1983).

${ }^{11}$ D. Goldstein, R. Handler, and L. Sirovich, "Direct numerical simulation of turbulent flow over a modeled riblet covered surface," Journal of Fluid Mechanics 302, 333 (1995).

${ }^{12}$ D. B. Goldstein and T.-C. Tuan, "Secondary flow induced by riblets," Journal of Fluid Mechanics 363, 115-151 (1998).

${ }^{13}$ O. A. El-Samni, H. H. Chun, and H. S. Yoon, "Drag reduction of turbulent flow over thin rectangular riblets," International Journal of Engineering Science 45, 436-454 (2007).

${ }^{14}$ C. Haecheon, P. Moin, and J. Kim, "Direct numerical simulation of turbulent flow over riblets," Journal of Fluid Mechanics 255, 503-539 (1993).

${ }^{15}$ Y. Suzuki and N. Kasagi, "Turbulent drag reduction mechanism above a riblet surface," AIAA Journal 32, 1781-1790 (1994).

${ }^{16}$ S. J. Lee and S. H. Lee, "Flow field analysis of a turbulent boundary layer over a riblet surface," Experiments in Fluids 30, 153166 (2001).

${ }^{17}$ R. García-Mayoral and J. Jiménez, "Hydrodynamic stability and breakdown of the viscous regime over riblets," Journal of Fluid Mechanics 678, 317-347 (2011).

${ }^{18}$ P. R. Viswanath, "Aircraft viscous drag reduction using riblets," Progress in Aerospace Sciences 38, 571-600 (2002).

${ }^{19}$ B. Dean and B. Bhushan, "Shark-skin surfaces for fluid-drag reduction in turbulent flow: a review," Philosophical transactions. Series A, Mathematical, physical, and engineering sciences 368 , 4775-4806 (2010).

${ }^{20}$ R. García-Mayoral and J. Jiménez, "Drag reduction by riblets," Philosophical transactions. Series A, Mathematical, physical, and engineering sciences 369, 1412-1427 (2011).

${ }^{21}$ R. Koeltzsch, K ., Dinkelacker, A., Grundmann, "Flow over convergent and divergent wall riblets," Experiments in Fluids 33, 346-350 (2002).

${ }^{22}$ H. Chen, F. Rao, X. Shang, D. Zhang, and I. Hagiwara, "Flow over bio-inspired 3D herringbone wall riblets," Experiments in Fluids 55, 1698 (2014).

${ }^{23}$ B. Nugroho, N. Hutchins, and J. P. Monty, "Large-scale spanwise periodicity in a turbulent boundary layer induced by highly ordered and directional surface roughness," International Journal of Heat and Fluid Flow 41, 90-102 (2013).

${ }^{24}$ B. Nugroho, Kevin, J. Monty, N. Hutchins, and E. Gnanaman- ickam, "Roll-modes generated in turbulent boundary layers with passive surface modifications," in 52nd Aerospace Sciences Meeting (American Institute of Aeronautics and Astronautics, Reston, Virginia, 2014).

${ }^{25}$ Kevin, J. P. Monty, H. L. Bai, G. Pathikonda, B. Nugroho, J. M. Barros, K. T. Christensen, and N. Hutchins, "Cross-stream stereoscopic particle image velocimetry of a modified turbulent boundary layer over directional surface pattern," Journal of Fluid Mechanics 813, 412-435 (2017).

${ }^{26} \mathrm{H}$. Q. Ho and M. Asai, "Experimental study on the stability of laminar flow in a channel with streamwise and oblique riblets," Physics of Fluids 30, 024106 (2018).

${ }^{27} \mathrm{~S}$. Tullis and A. Pollard, "The time dependent flow over V- and U-groove riblets of different sizes," Physics of Fluids 6, 1310-1314 (1994).

${ }^{28}$ Q. Liu, S. Zhong, and L. Li, "Reduction of pressure losses in a linear cascade using herringbone riblets," in Proceedings of ASME Turbo Expo 2017: Turbomachinery Technical Conference and Exposition (Charlotte, USA, 2017).

${ }^{29}$ B. Nugroho, V. Kulandaivelu, Z. Harun, N. Hutchins, and J. P. Monty, "An investigation into the effects of highly directional surface roughness on turbulent boundary layers," in 17th Australasian Fluid Mechanics Conference (Auckland, New Zealand, 2010).

${ }^{30}$ B. Nugroho, N. Hutchins, and J. P. Monty, "Effects of Diverging and Converging Roughness on Turbulent Boundary Layers," in 18th Australasian Fluid Mechanics Conference (Launceston, Australia, 2012).

${ }^{31}$ H. Chen, F. Rao, X. Shang, D. Zhang, and I. Hagiwara, "Biomimetic drag reduction study on herringbone riblets of bird feather," Journal of Bionic Engineering 10, 341-349 (2013).

${ }^{32}$ Kevin, B. Nugroho, J. P. Monty, and N. Hutchins, "Wall-Parallel PIV Measurements in Turbulent Boundary Layers with Highly Directional Surface Roughness," in 19th Australasian Fluid Mechanics Conference (Melbourne, Australia, 2014).

${ }^{33}$ R. Narasimha and S. N. Prasad, "Leading edge shape for flat plate boundary layer studies," Experiments in Fluids 17, 358360 (1994).

${ }^{34}$ J. J. Charonko and P. P. Vlachos, "Estimation of uncertainty bounds for individual particle image velocimetry measurements from cross-correlation peak ratio," Measurement Science and Technology 24, 065301 (2013).

${ }^{35}$ J. Zhou, R. J. Adrian, S. Balachandar, and T. M. Kendal1, "Mechanisms for generating coherent packets of hairpin vortices in channel flow," Journal of Fluid Mechanics 387, 353-396 (1999).

${ }^{36}$ S. Zhang and S. Zhong, "Experimental Investigation of Flow Separation Control Using an Array of Synthetic Jets," AIAA Journal 48, 611-623 (2010).

${ }^{37}$ F. Xu, Z. Gao, X. Ming, L. Xia, Y. Wang, W. Sun, and R. Ma, "The optimization for the backward-facing step flow control with synthetic jet based on experiment," Experimental Thermal and Fluid Science 64, 94-107 (2015).

${ }^{38}$ Y. Ma, R. Mohebbi, M. Rashidi, and Z. Yang, "Study of nanofluid forced convection heat transfer in a bent channel by means of lattice Boltzmann method," Physics of Fluids 30, 032001 (2018).

${ }^{39}$ R. Mohebbi, M. M. Rashidi, M. Izadi, N. A. C. Sidik, and H. W. Xian, "Forced convection of nanofluids in an extended surfaces channel using lattice Boltzmann method," International Journal of Heat and Mass Transfer 117, 1291-1303 (2018).

${ }^{40}$ R. Mohebbi, H. Lakzayi, N. A. C. Sidik, and W. M. A. A. Japar, "Lattice Boltzmann method based study of the heat transfer augmentation associated with $\mathrm{Cu} /$ water nanofluid in a channel with surface mounted blocks," International Journal of Heat and Mass Transfer 117, 425-435 (2018).

${ }^{41}$ R. Mejia-Alvarez, J. M. Barros, and K. T. Christensen, "Structural Attributes of Turbulent Flow Over a Complex Topography," in Coherent Flow Structures at Earth's Surface (John Wiley \& Sons, Ltd, Chichester, UK, 2013) pp. 25-41. 